\title{
EVALUATION OF SELF-INTERACTION PARAMETERS FROM BINARY PHASE DIAGRAMS
}

Thomas Lee Ellison

M.S. Thesis Submitted to Iowa State University

Ames Laboratory, ERDA

Iowa State University

Ames, Iowa 50011

Date Transmitted: October 1977

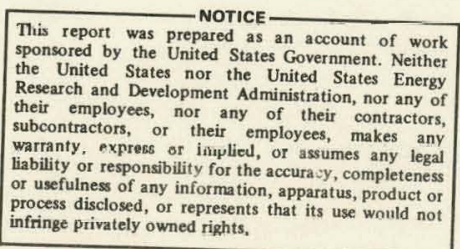

PREPARED FOR THE U.S. ENERGY RESEARCH AND DEVELOPMENT ADMINISTRATION UNDER CONTRACT NO. W-7405-eng-82 


\section{DISCLAIMER}

This report was prepared as an account of work sponsored by an agency of the United States Government. Neither the United States Government nor any agency Thereof, nor any of their employees, makes any warranty, express or implied, or assumes any legal liability or responsibility for the accuracy, completeness, or usefulness of any information, apparatus, product, or process disclosed, or represents that its use would not infringe privately owned rights. Reference herein to any specific commercial product, process, or service by trade name, trademark, manufacturer, or otherwise does not necessarily constitute or imply its endorsement, recommendation, or favoring by the United States Government or any agency thereof. The views and opinions of authors expressed herein do not necessarily state or reflect those of the United States Government or any agency thereof. 


\section{DISCLAIMER}

Portions of this document may be illegible in electronic image products. Images are produced from the best available original document. 
This report was prepared as an account of work sponsored by the United States Government. Neither the United States nor the United States Energy Research and Development Administration, nor any of their employees, nor any of their contractors, subcontractors, or their employees, makes any warranty, express or implied, or assumes any legal liability or responsibility for the accuracy, completeness, or usefulness of any information, apparatus, product or process disclosed, or represents that its use would not infringe privately owned rights.

Available from: National Technical Information Service U. S. Department of Commerce

P.O. Box 1553

Springfield, VA 22161

Price: Microfiche $\$ 3.00$ 


\section{Evaluation of selfinteraction parameters from binary phase diagrams}

by

Thomas Lee Ellison

A Thesis Submitted to the

Graduate Faculty in Partial Fulfillment of

The Requirements for the Degree of

MASTER OF SCIENCE

Department: Materials Science and Engineering

Major: Metallurgy

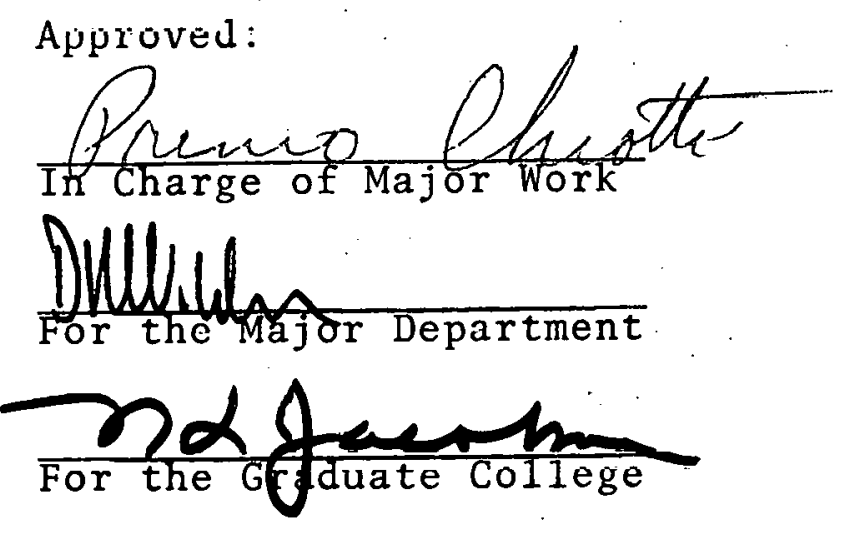

Iowa State University

Ames, Iowa 
TABLE OF CONTENTS

Abstract

I. : INTRODUCTION

Page

1

II . LITERATURE REVIEW

A. Interaction Parameters 6

B. Relationship between Henry's Law and 16

C. The Calculation of Thermodynamic $\quad 22$ Properties from Phase Diagrams

III. METHODS OF DETERMINING SELF-INTERACTION । 32

PARAMETERS FROM EXPERIMENTAL DATA.

A. Determination from Activity Coefficient 32 Measurements

B. Determination from Thermodynamic Data 37

C. Determination Directly from Binary Phase.. 40 Diagrams

IV. RESULTS AND DISCUSSION 60

V. SUMMARY 77

VI . LITERATURE CITED 79

VII. ACKNOWLEDGEMENTSS $\quad 84$

VIII. APPENDIX $\quad 85$

A. Derivation of Equation I-10 85

B. Derivation of Equations II-38 and II-39 90 


\section{Evaluation of self-interaction parameters \\ from binary phase diagrams**}

\section{Thomas Lee E1lison}

The feasibility of calculating Wagner self-interaction parameters from binary phase diagrams was examined. The selfinteraction parameters of 22 non-ferrous liquid solutions were calculated utilizing an equation based on the equality of the chemical potentials of a component in two equilibrium phases. Utilization of the equation requires the evaluation of the first and second derivatives of various liquidus and solidus data at infinite dilution of the solute component. Several numerical methods for evaluating the derivatives of tabular data were examined. A method involving power series curve fitting and subsequent differentiation of the power series was found to be the most suitable for the interaction parameter calculations. Comparison of the calculated self-interaction parameters with values obtained from thermodynamic measurements indicates that the Wagner self-interaction parameter can be successfully calculated from binary phase diagrams.

*USERDA Report IS-T-772. This work was performed under Contract W-7405-eng-82 with the Energy Research and Development Administration. 


\section{INTRODUCTION}

Most materials, whether by accident or design, are composed of several components. In fact, the concept of a pure substance is more an ideal than a reality since even the purest of materials contain some foreign constituents. Whether comprised of a single phase or a mixture of phases, almost all materials can be considered to be solutions. Since the presence of even minute impurities can have a drastic effect on the properties of metals, the ability to understand, predict, and control the behavior of solutions is of immense importance to the metallurgist. Although such understanding must ultimately come from fundamental atomic and statistical theory, thermodynamics can, in the meantime, serve as a useful tool in describing and predicting the behavior of solutions.

The thermodynamic properties of a binary solution may be described in terms of the change in the Gibbs free energy upon mixing two components to form a homogeneous solution at constant temperature and pressure. This change is known as the free energy of mixing, $\Delta G_{m}$, and is given by the relation

$$
\Delta G_{m}=x_{1} \Delta \bar{G}_{1}+x_{2} \Delta \bar{G}_{2} \text {. }
$$

If more than two components are involved,

$$
\Delta G_{m}=\sum_{i} x_{i} \Delta \bar{G}_{i}
$$

In Equations $I-I$ and $I-2, \Delta \bar{G}_{i}$, the relative partial molar free 
energy, is defined as

$$
\Delta \bar{G}_{i}=\bar{G}_{i}-G_{i}^{o}=\operatorname{RT} \ln a_{i}=\operatorname{RT} \ln \gamma_{i} x_{i}
$$

where $\bar{G}_{i}$ is the partial molar free energy, $\left(\partial G^{\prime} / \partial n_{i}\right)_{T}, P, n_{j}$; $G_{i}^{0}$ is the free energy of component $i$ in $i$ ts standard state (usually the pure component in the same state of aggregation as the solution in question); $R$ is the gas constant, 1.987 cal $\cdot \operatorname{mole}^{-1} \cdot \mathrm{K}^{-1} ; \mathrm{T}$ is the temperature in ${ }^{\circ} \mathrm{K} ; \mathrm{a}_{i}$ is the activity; $x_{i}$ is the mole fraction of component $i$; and $\gamma_{i}$ is the activity coefficient. For a binary system, if $\Delta \bar{G}_{1}$ is known, $\Delta \widehat{G}_{2}$ can be obtained from the Gibbs-Duhem equation,

$$
\overline{\mathrm{x}}_{1} \mathrm{~d} \Delta \overline{\mathrm{G}}_{1}+\overline{\mathrm{x}}_{2} \mathrm{~d} \Delta \overline{\mathrm{G}}_{2}=0
$$

by using various graphical and analytical integration techniques.

The relative partial molar free energy, $\Delta \bar{G}_{\dot{i}}$, is a function of temperature, pressure, and composition. For the hypothetical ideal solution, the functional dependence of $\Delta \bar{G}_{i}$ can be expressed as

$$
\Delta \overline{\mathrm{G}}_{\mathbf{i}}^{\mathrm{IDEAL}}=\operatorname{RT} \ln \mathrm{x}_{\mathbf{i}}
$$

For real systems, however, no expression for $\Delta \bar{G}_{i}=f\left(T, P, x_{i}\right)$ of general applicability has been found. As a result, real solutions are often described in terms of an ideal part and

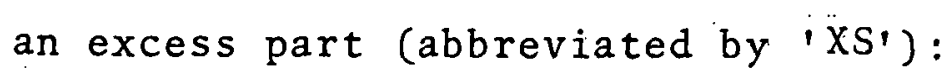

$$
\Delta \bar{G}_{i}=\Delta \bar{G}_{i}^{I D E A L}+\Delta \bar{G}_{i}^{X S}=\operatorname{RT} \ln x_{i}+R T \ln \gamma_{i}
$$


Some of the functional dependence of $\Delta \bar{G}_{i}$ is thus transferred to $\Delta \bar{G}_{i}^{X S}$ :

$$
\Delta \bar{G}_{i}^{X S}=R \operatorname{Rin} \gamma_{i}=f\left(T, P, x_{2}, x_{3} \ldots\right)
$$

For a binary system at constant pressure, $\Delta \bar{G}_{i}^{X S}$ may be considered to be a function of only two variables:

$$
\Delta \bar{G}_{\dot{\mathrm{I}}}^{\mathrm{XS}}=\mathrm{f}\left(\mathrm{T}, \mathrm{x}_{\dot{1}}\right)
$$

The analytical representation of the relative partial molar quantities $\Delta \mathrm{G}_{\mathrm{m}}$ and $\Delta \overline{\mathrm{G}}_{\dot{\mathrm{I}}}^{\mathrm{XS}}$ have been the subject of continual investigation in metallurgical thermodynamics for over fifty years. Numerous mathematical formalisms have been developed in an attempt to organize the experimental data and to facilitate calculations using these data. The bases for these formulations range from statistical thermodynamic models to equations based solely on empirical relationships.

One of these formalisms, the interaction parameter representation, has gained increasing popularity over the past several decades as a means of representing dilute solution data. The interaction parameter representation is a constant temperature, constant pressure Taylor's series expansion of $\ln \gamma_{2}=f\left(x_{2}, x_{3} \ldots\right)$ about the point $x_{2}, x_{3} \ldots=0$ $\left(x_{1}=1\right)$ where $x_{2}, x_{3} \ldots$ represent various solutes in a common solvent $x_{1}$. The coefficients of this expansion are called interaction parameters and they represent the various partial derivatives of $1 n \gamma_{2}$ in the Taylor's series. The self-inter- 
action parameter, $\varepsilon_{\dot{1}}^{\dot{1}}$, is the coefficient of the second term of this expansion and is defined by

$$
\varepsilon_{i}^{\dot{i}}=\left(\frac{\partial \ln \gamma_{i}}{\partial x_{i}}\right)_{\ddot{x}_{i} \rightarrow 0}
$$

where $i$ refers to a solute dissolved in a solvent 1 .

Values for the interaction parameters are usually obtained from the slopes of experimentally determined $1 \mathrm{nr}_{\mathrm{i}}$ versus $x_{i}$ curves. It is often, however, difficult to obtain activity coefficient data of sufficient accuracy and extent to permit the precise calculation of these parameters. The phase diagram of a system is intimately related to its thermodynamic properties and can, in many cases, be used to obtain. this much needed information. Chiotti. (7) has derived an equation, similar to one by Petot-Ervas et al. (47), which allows the calculation of self-interaction parameters from binary phase boundary data. Letting 1 represent the solvent and 2 represent the solute, the equation is given by:

$$
\begin{aligned}
\varepsilon_{2}^{2}= & \left(\frac{\mathrm{d}^{2} \ln \frac{\mathrm{x}_{1}^{\prime}}{\mathrm{x}_{1}}}{\mathrm{~d} \mathrm{x}_{1}^{\prime 2}}\right)_{\mathrm{x}_{1}^{\prime} \rightarrow 1}+\frac{2}{\mathrm{~T}_{\mathrm{f}_{1}}}\left(\frac{\mathrm{d} \ln \frac{\mathrm{x}_{1}^{\prime}}{\mathrm{x}_{1}^{\prime \prime}}}{\mathrm{d} \mathrm{x}_{1}^{\prime}}\right)_{\mathrm{x}_{1}^{\prime}+1}\left(\frac{\mathrm{d}^{\prime} \mathrm{T}}{\mathrm{d} \mathrm{x}_{1}^{\prime}}\right)_{\mathrm{x}_{1}^{\prime}+1} \\
& -\frac{\Delta s_{f_{1}}^{0}}{\mathrm{RT}_{\mathrm{f}_{1}}}\left(\frac{\mathrm{d}^{2} \mathrm{~T}}{\mathrm{dx_{1 } ^ { \prime 2 }}}\right)_{\mathrm{x}_{1}^{\prime}+1}
\end{aligned}
$$

The superscripts (') and (") represent the liquidus and the 
solidus, respectively, and $\mathrm{T}_{f_{1}}$ and $\Delta S_{f_{1}}^{o}$ are the temperature and entropy of fusion. The derivatives are obtained from liquidus and solidus data and are to be evaluated at $x_{i}^{\prime}=1.0$ $\left(x_{2}^{\prime}=0\right)$. The equation is based on the equality of the chemical potentials of a component in two equilibrium phases and can be applied to any liquidus-solidus phase diagram possessing limited or negligible solid solubility.

The purpose of this investigation is to evaluate the determination of self-interaction parameters from binary phase diagrams. The se1f-interaction parameters of 22 binary solutions will be calculated from the derivatives of their liquidus and solidus curves utilizing Equation I-10. Various numerical methods for evaluating the derivatives will be investigated and the results compared with values obtained from dilute solution thermodynamic measurements. 


\section{II. : LITERATURE REVIEW}

\section{A. Interaction Parameters}

Various formulations have been proposed over the past century to analytically describe the thermodynamic properties of binary solutions. In 1895, Margules (42) suggested the use of a power series polynomial to represent the activity coefficients of binary alloys. Several years later, van Laar (60) introduced a solution equation based on the van der Waals equation for gases. In 1929, Hildebrand proposed the popular "regular solution" model. Since that time, many equations of both theoretical and empirical origin have been developed in an attempt to relate the thermodynamic properties of solutions to composition and temperature. Various relations have been developed by Guggenheim (18, p. 196) (quasichemical mode1), Scatchard and Hamer (51), Lumsden (34), Hardy (22) (subregular solution mode1), Krupkowski (31), Wagner (62, pp. 51-53) (interaction parameters), Wilson (66), Darken (10) (quadratic formalism), and other authors. Many of these expressions lack the flexibility to allow their application to the broad range of properties exhibited by metallic solutions. Because of this, several investigators $(65,1,2)$ have recently advocated the use of empirically based Fourier and Legendre series representations as a means of obtaining analytical expressions for use in computer calculations.

Most alloys of metallurgical interest involve four or 
more dilute solutes dissolved in a common solvent. The majority of the existing analytical expressions apply to binary systems and cannot be utilized in situations where more than two solutes are present. Most of the existing analytical equations possess either a firm theoretical basis with a limited scope of application or a wide scope of application with very little relation to physical models. However, for dilute solutions, all of these criteria can be satisfied by the Wagner interaction parameter representation (62, pp. 5153). The Wagner expression is applicable to a wide variety of experimental data and it can be easily extended to represent multicomponent solutions. In addition to its ease of empirical application, the experimentally determined interaction parameters can be interpreted in terms of interactions between solute atoms. Because of these desirable qualities, the interaction parameter representation has gained considerable usage over the past few years as a mathematical description of dilute solution data.

The interaction parameter representation was introduced by Wagner $(62, \mathrm{pp} .51-53)$ in 1952 in an attempt to describe the thermodynamic properties of dilute multicomponent solutions. He suggested that a Taylor's series expansion of $\ln \gamma_{2}=$ $f\left(x_{2}, x_{3} \ldots\right)$ about the point $x_{i}=0$ be user to represent the activity coefficients of dilute solutes dissolved in a common solvent: 


$$
\begin{aligned}
& \ln \gamma_{2}\left(x_{2}, x_{3} ; x_{4} \ldots\right)=\ln \gamma_{2}^{0}+\left[x_{2} \frac{\partial \ln \gamma_{2}}{\partial x_{2}}+x_{3} \frac{\partial \ln \gamma_{2}}{\partial x_{3}}+\ldots\right] \\
& +\left[\frac{1}{2} x_{2}^{2} \frac{\partial^{2} \ln \gamma_{2}}{\partial x_{2}^{2}}+x_{2} x_{3} \frac{\partial^{2} \ln \gamma_{2}}{\partial x_{2} \partial x_{3}}+\ldots\right]+\ldots
\end{aligned}
$$

The subscripts $2,3,4, \ldots$ represent the various solute species dissolved in the solvent 1 and $\gamma_{2}^{0}$ is the activity coefficient of solute 2 at infinite dilution $\left(x_{2}=0\right)$. All of the partial derivatives are to be evaluated at $x_{i}=0$. By defining the partial derivatives in the following manner:

$\varepsilon_{2}^{2} \equiv\left(\frac{\partial \ln \gamma_{2}}{\partial x_{2}}\right)_{x_{2}=0}, \varepsilon_{2}^{3} \equiv\left(\frac{\partial \ln \gamma_{2}}{\partial x_{3}}\right)_{x_{3}=0}$, or in general $\varepsilon_{i}^{j}=\left(\frac{\partial \ln \gamma_{i}}{\partial x_{j}}\right)_{x_{j}=0}$ and by ignoring the higher order terms of the expansion, Wagner stated that the expansion could be easily represented by the expression:

$$
\ln \gamma_{2}=\ln \gamma_{2}^{o}+x_{2} \varepsilon_{2}^{2}+x_{3} \varepsilon_{2}^{3}+x_{4} \varepsilon_{2}^{4}+\ldots
$$

He further noted that for practical calculations, Equation II- 2 could be written in terms of weight percent and common logarithms :

$$
\log \gamma_{2}=\log \gamma_{2}^{0}+e_{2}^{2}\left(\frac{8}{0} 2\right)+e_{2}^{3}\left(\frac{8}{2} 3\right)+e_{2}^{4}\left(\frac{8}{0} 4\right)+\ldots
$$

Using the Gibbs-Duhem equation, Wagner also derived the following reciprocity relationship for ternary interaction 
parameters :

$$
\varepsilon_{j}^{i}=\varepsilon_{i}^{j}
$$

Several years after the introduction of interaction parameters, Chipman (9) utilized this representation to describe the effects of several solutes in steel. In 1960 , Schenck, Frohberg and Steinmetz (52) presented the following expression for converting the weight percent interaction parameter, $e_{i}^{j}$, to the atomic fraction parameter, $\varepsilon_{i}^{j}$ :

$$
\varepsilon_{i}^{j}=230 \frac{M_{j} e_{i}^{j}}{M_{1}}+\frac{M_{1}-M_{j}}{M_{1}}
$$

$M_{j}$ is the atomic weight of solute $j$ and $M_{1}$ is the atomic weight of the solvent. In 1963, Dealy and Pehlke (11) summarized the existing non-ferrous dilute solution data in terms of interaction parameters. They also discussed the temperature relationships for the interaction parameters, recommending that a. linear dependence on $1 / \mathrm{T}$ be used for extrapolation or interpolation of $\varepsilon_{i}^{j}$.

In 1965, Lupis and El1iott (38) extended the interaction parameter formalism to include entropy and enthalpy interaction coefficients:

$$
\delta_{i}^{j} \equiv\left(\frac{\partial S_{i}^{X S}}{\partial x_{j}}\right)_{x_{1} \rightarrow 1} \text { and } \eta_{i}^{j} \equiv\left(\frac{\partial H_{i}^{M}}{\partial x_{j}}\right)_{x_{1} \rightarrow 1} \text {. }
$$

These coefficients are related to the free energy interaction parameter by the following relation: 


$$
\varepsilon_{i}^{j}=\frac{n_{i}^{j}}{R T}-\frac{\delta_{i}^{j}}{R}
$$

In subsequent papers $(39,40)$, Lupis and Elliott developed a "generalized" formulation of the Taylor's series expansion which would allow the application of the interaction parameter concepts to non-dilute solutions. They suggested that the higher order terms of the Taylor's series expansion be used when attempting to describe solutions in the non-dilute region. They proposed the following formulation for representing the activity coefficient of a solute species in a multicomponent solution:

$$
\text { In } \frac{\gamma_{i}}{\gamma_{i}^{o}}=\sum_{j=2}^{m} \varepsilon_{i}^{j} x_{j}+\sum_{j=2}^{m} \rho_{i}^{j} x_{j}^{2}+\sum_{j=2}^{m} \sum_{\substack{k=2 \\ j \neq k}}^{m} \rho_{i}^{j, k} x_{j} x_{k}(I I-7)
$$

where $\rho_{i}^{j} \equiv \frac{1}{2}\left(\frac{\partial^{2} 1 n \gamma_{i}}{\partial x_{j}^{2}}\right)_{x_{1} \rightarrow 1}$ and $\rho_{i}^{j, k} \equiv\left(\frac{\partial^{2} 1 n \gamma_{i}}{\partial x_{j} \partial x_{k}}\right)_{x_{1} \rightarrow 1}$.

For a ternary solution, Equation II-7 would simplify to the following:

$$
\ln \dot{\gamma}_{2}=\ln \gamma_{2}^{0}+\varepsilon_{2}^{2} x_{2}+\varepsilon_{2}^{3} x_{3}+\rho_{2}^{2} x_{2}^{2}+\rho_{2}^{2,3} x_{2} x_{3}+\rho_{2}^{3} x_{3}^{2}
$$

The parameters may be obtained by either graphically or analytically evaluating the limiting slopes of various $1 \mathrm{n}^{\gamma}{ }_{i}$ versus composition curves. Sigwurth and Elijott (56) have compiled a fairly recent summary of experimental first and second order interaction parameters for solutes in liquid 
iron. The cross-product second order terms, $\rho_{i}^{j, k}$, are generally not in use since there is little experimental data accurate enough to permit their calculation.

Several statistical solution models have been developed to interpret the interaction parameters of Equation II-7 in terms of atomic interactions. Lupis and Elliott (40) extended Guggenheim's quasi-chemical approximation to multicomponent systems, and then used the model to predict several first and second order interaction parameters. They found the agreement between the experimental values and those of the model to be only fair. Lupis and Elliott suggested that this lack of agreement was the result of the inadequacy of several assumptions of the quasi-chemical model. In the quasi-chemical model, it is assumed that the vibrational contribution to the excess entropy can be neglected, It is further assumed that the bond energy. between two atoms is independent of the surroundings. In order to overcome these limitations, Lupis and Elliott (41) developed the "central atoms" mode1. This model is based on the configurational changes of nearest-neighbor shells surrounding a central atom and can be applied both to disordered solids and liquid solutions. For the liquid state, an expression from the Eyring ceil model (14) is used to describe the partition functions of the central atoms model. The probabilities of various shell configurations about the central atom are examined and 
the overall properties of the solution are obtained by summing the contributions of each atom configuration.

Lupis (37), in a rather noteworthy contribution on the thermodynamic formalisms of metallic solutions, has given a qualitative interpretation of the atomistic significance of interaction parameters. A summary of his interpretation is given in Table $1 . \quad \ln \gamma_{B}^{\circ}$, for example, compares the energy of a solute atom, $B$, surrounded by $Z$ solvent atoms. Similarly, $\varepsilon_{B}^{B}$ represents a configuration of one $B$ atom surrounded by another $B$ solute atom and Z-1 atoms of solvent, $A$. An examination of the configurations of $\varepsilon_{B}^{C}$ and $\varepsilon_{C}^{B}$ reveals that they are physically equivalent; this is consistent with Equation II-4. For very dilute solutions, the probability of two $B$ atoms surrounding a third B atom would become very small. It can be seen from Equation II- 8 that the second order terms do indeed become negligible in the dilute region since $x_{B}^{2}$ approaches zero much more rapidly than $x_{B}$. Likewise, the contribution of the $\rho_{B}^{(B, C)}$ term should depend on concentrations of both $B$ and $C$ atoms and the $\rho_{B}^{(B, C)} x_{C} x_{B}$ term in Equation II8 does depend on both concentrations.

Darken (10) has criticized the interaction parameter approach, claiming that it is mathematically inconsistent with classical thermodynamics at finite concentrations. Using the Gibbs-Duhem equation for ternary systems, he derived the following expression which must be satisfied by any ternary 
Table 1. Qualitative atomistic interpretation of the interaction parameters of a dilute ternary solution composed of solutes $B$ and $C$. in a solvent $A^{a}$

\begin{tabular}{|c|c|c|c|}
\hline \multirow{2}{*}{$\begin{array}{l}\text { Interaction } \\
\text { parameter }\end{array}$} & \multirow{2}{*}{$\begin{array}{l}\text { Species comprising } \\
\text { the central atom }\end{array}$} & \multicolumn{2}{|c|}{ Surrounding atoms } \\
\hline & & Number of & Species \\
\hline $\ln \gamma_{B}^{o}$ & B & $z^{\mathrm{b}}$ & A \\
\hline$\varepsilon_{\mathrm{B}}^{\mathrm{B}}$ & B & $\begin{array}{c}1 \\
z-1\end{array}$ & $\begin{array}{l}\text { B } \\
\text { A }\end{array}$ \\
\hline$\varepsilon_{B}^{C}$ & B & $\begin{array}{c}1 \\
z-1\end{array}$ & $\begin{array}{l}\text { C } \\
\text { A }\end{array}$ \\
\hline$\varepsilon_{\mathrm{C}}^{\mathrm{B}}$ & C. & $\begin{array}{c}1 \\
z-1\end{array}$ & $\begin{array}{l}\mathrm{B} \\
\mathrm{A}\end{array}$ \\
\hline$\rho_{B}^{B}$ & $B$ & $\underset{z-2}{2}$ & $\begin{array}{l}\mathrm{B} \\
\mathrm{A}\end{array}$ \\
\hline$\rho_{B}^{(B, C)}$ & B & $\begin{array}{c}1 \\
1 \\
z-2\end{array}$ & $\begin{array}{l}\text { B } \\
\text { C } \\
\text { A }\end{array}$ \\
\hline
\end{tabular}

$a_{\text {This }}$ table was derived from an atomistic interpretation of the "central atoms" theory given by Lupis (37). she 11 .

$b_{Z}$ is the coordination number of the nearest neighbor

mathematical expression if it 1 s to be thermodynamically consistent:

$$
\left(1-x_{3}\right) \frac{\partial \ln \gamma_{2}}{\partial x_{3}}+x_{3} \frac{\partial \ln \gamma_{3}}{\partial x_{3}}=x_{2} \frac{\partial \ln \gamma_{2}}{\partial x_{2}}+\left(1-x_{2}\right) \frac{\partial \ln \gamma_{3}}{\partial x_{2}}
$$

Using only the first order terms of Equation II-8, he demonstrated that Equation II-9 becomes:

$$
\left(1-x_{3}\right) \varepsilon_{2}^{3}+x_{3} \varepsilon_{3}^{3}=x_{2} \varepsilon_{2}^{2}+\left(1-x_{2}\right) \varepsilon_{3}^{2}
$$


Since Equation (II-10) cannot be satisfied at finite concentrations, Darken proposed the "quadratic formalism" to serve as an alternate expression for dilute solutions. The quadratic formalism is based on the following relations:

$$
\begin{aligned}
& \log \gamma_{1}=\alpha_{12}\left(1-x_{1}\right)^{2} \\
& \log \gamma_{2}=\log \gamma_{2}^{0}-2 \alpha_{12} x_{2}+\alpha_{12} x_{2}^{2}
\end{aligned}
$$

where the subscript 1 represents the solvent and the subscript 2 represents the solute.

Lupis. (36) has countered Darken's criticism by stating that the second order terms should not have been neglected when applying Equation II-9. When the second order terms are included, Equation II-9 becomes:

$$
\rho_{2}^{2,3}-\rho_{3}^{3,2}=2 \rho_{3}^{2}-2 \rho_{2}^{3}+\varepsilon_{2}^{2}-\varepsilon_{3}^{3}
$$

The following relations may be obtained by applying the GibbsDuhem equation to the ternary interaction parameter representations (see Equation II-8) of $\ln \gamma_{2}$ and $\ln \gamma_{3}$ :

$$
\begin{aligned}
& \rho_{2}^{2,3}+\varepsilon_{2}^{3}-2 \rho_{3}^{2}+\varepsilon_{2}^{2} \\
& \rho_{3}^{3,2}+\varepsilon_{3}^{2}=2 \rho_{2}^{3}+\varepsilon_{3}^{3}
\end{aligned}
$$

Equation II-13 may be obtained by subtracting. Equation II-15 from Equation II-14 and noting that $\varepsilon_{2}^{3}=\varepsilon_{3}^{2}$. Thus, when the second order terms are included, no inconsistency is present. Lupis further noted that the quadratic formalism is itself a 
polynomial representation. Unlike the interaction parameter representation; the polynomial degree of Darken's formalism is chosen a priori rather than allowing the expression to vary with experimental conditions.

In the same paper, Lupis also developed guidelines for the application of the interaction parameter representation to experimental data. He derived several relations which can be used to determine the concentration limits of the interaction parameter polynomial. The general Taylor's series can be divided into two parts:

$$
Y\left(x_{2}\right)=Y_{n}\left(x_{2}\right)+R_{n}\left(x_{2}\right)
$$

where $\mathrm{n}$ is the polynomial degree, and $R_{n}\left(x_{2}\right)$ is the remainder. Since $0 \leq x_{2} \leq 1, R_{n}$ decreases as $n$ increases. In order to obtain an adequate representation of the data

$$
\left|R_{n}\right|<\delta Y
$$

where $\delta Y$ is the experimental error in the $1 n \gamma_{i}$ values: A polynomial of sufficient degree, $n$, must be selected which will insure that Equation II-17 holds. As was mentioned earlier, at present, the data are barely accurate enough to justify the use of second order terms $(n=2)$. Lupis also presents several other conditions based on stability considerations which can be used to help determine the range of application of the Taylor's series:

$$
\text { if } \ln \gamma_{2}=\ln \gamma_{2}^{\circ}+\varepsilon_{2}^{2} x_{2} \text {, then } \varepsilon_{2}^{2} \geq-1 / x_{2} \text { : }
$$


if $\ln \gamma_{2}=\ln \gamma_{2}^{o}+\dot{\varepsilon}_{2}^{2} x_{2}+\rho_{2}^{2} x_{2}^{2}$, then

$$
1+\varepsilon_{2}^{2} x_{2}+2 \rho_{2}^{2} x_{2}^{2} \geq 0
$$

B. Relationship between Henry's Law and Interaction Parameters

Since Henry's law is often applied to the dilute solution region, its effect on the interaction parameter representation should be considered. Henry's law has traditionally implied the existence of a proportionality between the activity of a solute and its concentration in the dilute solution region. Several interpretations of Henry's law exist and each differs in the degree of strictness in which the proportionality should be applied. Some authors (16, pp. 337-340; 23, pp. 315317) suggest that the proportionality strictly holds over an infinitesimal composition fnterval near $x_{2}=0$ known as the infinitely dilute region. Others $(12, \mathrm{p} .225 ; 35)$, contend that the proportionality exists only as an approximation and that the frequently used Henry's law-line of activity plots is merely the limiting slope of the activity curve at $x_{2}=0$. This dis tinction is largely academic when considering activity curves, however, several conflicting conclusions arise when other thermodynamic properties are considered.

In Pitzer and Brewer's revision of the text Thermodynamics by Lewis and Randall (33, pp. 231-239), a "proof" has been given that Henry's law is strictly proportional over an 
infinitesimal interval near $x_{2}=0$. The authors begin by assuming that $\left(\mathrm{df}_{2} / \mathrm{dx}_{2}\right)_{\mathrm{x}_{2}=0}$ is finite, $\mathrm{f}_{2}$ being the fugacity of the solute. They state that, in the immediate neighborhood of a point, where $x=0$ and $y=0$, it can be demonstrated from elementary calculus that $d y / d x=y / x$. Noting that $f_{2}=0$ when $x_{2}=0$, the authors obtain the following relations for the infinitely dilute region:

$$
\frac{\partial f_{2}}{\partial x_{2}}=\frac{f_{2}}{x_{2}}=\text { constant }
$$

or

$$
\mathrm{f}_{2^{i}}=\mathrm{kx}_{2}
$$

In their discussion of the implications of Equations II-20 and II-21, the authors state that the $f_{2}$ versus $x_{2}$ curve "... starts out as a straight line, and in the infinitely dilute solution the fugacity of the solute is proportional to its mole fraction." They further assert that this proportionality strictly holds in the infinitely dilute region and that Equation II-21 is valid for all solutions regardless of how many solute species are present.

Their discussion implies that, in some interval, $x_{2}=$ $0+\delta x$, the fugacity of the solute is strictly equal to a constant, $k$, times the mole fraction of the solute. The consequences of this conclusion are severe with regards to the interaction parameter approach. The activity coefficient of the solute, $\gamma_{2}$, can be related to the Henry's law constant, 
$\mathrm{k}$, by the relation

$$
r_{2}=\frac{f_{2}}{f_{2}^{o} x_{2}}=\frac{k}{f_{2}^{0}}=k^{\prime}
$$

where $f_{2}^{o}$ is the fugacity of the pure solute, a constant. If $k$ is constant over some interval $x_{2}=0+\delta x$, then $\gamma_{2}$ and 1 in $\gamma_{2}$ will also be constant over this same interval. As a result, the $\ln \gamma_{2}$ versus $x_{2}$ curve must have zero slope over the interval $x_{2}=0+\delta x$. Since the interaction parameters are defined as the derivatives of $\ln \gamma_{2}$ with respect to $x_{2}$ at $x_{2}=0$, $\varepsilon_{2}^{2}$ and all higher order interaction parameters will be zero. These conclusions cannot be justified on an empirical basis. Numerous determinations of the interaction parameter have demonstrated that the slope of the $\ln \gamma_{2}$ curve is, in general, very different from zero. Lupis (35), for example, has presented a summary plot of $\ln \gamma_{2}$ versus $x_{2}$ curves for eight typical metal systems. The data range from $x_{2}=0.01$ to $x_{2}=0.001$ atomic fraction and some curves have as many as 20 carefully determined points. All of the limiting slopes are clearly nonzero and a few of the slopes are quite large. Since all $1 \mathrm{nr}_{2}$ measurements must be made at finite concentrations, it would, admittedly, be difficult, if not impossible to experimentally prove that the true limiting slopes are nonzero. Nevertheless, the extrapolated empirical slopes indicate that the $\ln \gamma_{2}$ curves, in general, trend toward nonzero slopes. 
In addition to this experimental evidence, a careful examination of the origin of Equation II-20 indicates that the strict proportionality interpretation also has no mathematical basis. The calculus "proof" of Lewis and Randall, when critically examined, indicates that Equations II-20 and II-21 should be viewed as approximations rather than strict equalities. Equation II-20 may be obtained by representing the fugacity by a Taylor's series expansion of the function $f_{2}=f_{2}\left(x_{2}\right)$ in some neighborhood of the point $x_{2}=0$ :

$$
f_{2}\left(x_{2}\right)=\sum_{i=0}^{\infty} \frac{f_{2}^{(i)}(b)}{i !}\left(x_{2}-b\right)^{i}
$$

Since the point of expansion, $b$, is zero and since $f_{2}(0)=0$, Equation II-23 becomes:

$$
f_{2}\left(x_{2}\right)=f_{2}^{\prime}(0) x_{2}+\frac{1}{2} f_{2}^{\prime \prime}(0) x_{2}^{2}+\frac{1}{3} f_{2}^{\prime \prime \prime}(0) x_{2}^{3}+\ldots
$$

If, in the immediate neighborhood of the point $x_{2}=0$, the $\frac{1}{2} f_{2}^{\prime \prime}(0) x_{2}^{2}$ term and al1 higher order terms are ignored, Equation II -24 becomes:

$$
f_{2}\left(x_{2}\right)=f_{2}^{\prime}(0) x_{2}=\left(\frac{d f_{2}}{d x_{2}}\right)_{x_{2}=0} x_{2}
$$

which upon rearrangement gives Equation II -20:

$$
\left(\frac{d f_{2}}{d x_{2}}\right)_{x_{2}=0}=\frac{f_{2}}{x_{2}}=a \text { finite value. }
$$

The higher order terms of Equation II-24 must be ignored in order to obtain Equation II-20. If the terms are deleted be- 
cause $f_{2}^{\prime \prime}(0)$ and all higher derivatives are assumed to be zero, then Equation II-20 cannot serve as a proof that $f_{2}$ is linear over some interval, since such an assumption would a priori require the function to be linear. Alternately, if the terms are ignored on the basis that they are negligible, in comparison to $f_{2}^{\prime}(0) x_{2}$, then Equations II-20 and II-2I must be viewed as approximations. In any case, Equations II-20 and II-21 cannot be considered as proof that $f_{2}=k x_{2}$ is a strict equality over some interval.

While it would be difficult to conclusively prove that the interval interpretation of Henry's law is incorrect, this interpretation cannot, at present, be justified on either an empirical or a mathematical basis, In general, Henry's law should be considered as an approximation rather than a strict proportionality, unless specific experimental evidence indicates otherwise. Although activity curves often appear to be linear near $x_{2}=0$, the existence of a slight curvature should be considered as a possibility. Exponential curves, for example, often possess regions where the curves are, for most purposes, linear, yet the curves still have a small curvature and nonzero derivatives in this region. While this distinction between approximation and strict obedience is somewhat trivial when considering activity curves, the effects of a slight curvature can be detected in carefully determined $\ln \gamma_{2}$ plots. 
Lupis (35) has presented a more precise statement of Henry's law which is consistent with the interaction parameter approach. Lupis has cast the phenomena of Henry's 1aw and Raoult's law in terms of limiting values and slopes. He defines Henry's law as being obeyed when $\left(\mathrm{da}_{2} / \mathrm{dx}_{2}\right)_{\mathrm{x}_{2} \rightarrow 0}$ is equal to a finite, nonzero value. In reference to the activity coefficient, Lupis divides this statement of Henry's law into two cases. The first case, which he calls Henry's zeroth order law, states that the value of $\ln \gamma_{2}^{0}$ is finite. The second case, Henry's first order law, states that both 1 in ${ }_{2}^{0}$ and $\left[\left(\mathrm{dln}_{2}\right) /\left(d x_{2}\right)\right]_{x_{2} \rightarrow 0}$ are finite values. According to Lupis, the zeroth order law may be interpreted in terms of correct selection of the species describing the system. A correct choice of reacting species will yield a finite value for $1 n Y_{2}^{\circ}$ while an incorrect choice will lead to an infinite value. He states that the first order law may be interpreted in terms of the short-range effectiveness of forces in solutions. This interpretation is not surprising since $\left[\left(\mathrm{d}^{\mathrm{n}} \gamma_{2}\right) /\right.$ $\left.\left(d x_{2}\right)\right]_{x_{2} \rightarrow 0}$ is the definition of the self-interaction parameter, $\varepsilon_{2}^{2}$. Lupis makes the observation that the zeroth order law is obeyed by both electrolytic and nonelectrolytic solutions. The first order law, however, is primarily only obeyed by nonelectrolytes such as metallic solutions. Although this definition of Henry's law is considerably different from the statements traditionally used, Lupis contends that such a 
definition is a necessity if Henry's law is truly to be considered a law and not merely an approximation.

C. The Calculation of Thermodynamic Properties from Phase Diagrams

The thermodynamic properties of a binary system are closely related to its temperature-composition phase diagram. In the absence of surface or field effects, any of the molar properties such as $\Delta G_{m}, \Delta \bar{G}_{i}$, or $\Delta \bar{H}_{i}$ can be considered to be a function of temperature, pressure, and composition: $Y=$ $Y\left(T, P, x_{i}\right)$ where $Y$ is some arbitrary molar quantity. If the pressure is held constant, $Y$ becomes a function of two variables, $T$ and $x_{i}$, and the functional dependence of $Y$ can then be visualized as a collection of surfaces in three dimensional $Y-T-x_{i}$ space. Each surface denotes the equilibrium functional dependence of $Y$ for some phase or mixture of phases (59, pp. 164-177). When the intersections of these surfaces are projected onto the $T-x_{i}$ plane, the familiar phase diagram, commonly used in metallurgy, is produced (59, pp. 164-177). The $\mathrm{T}-\mathrm{x}_{\mathrm{i}}$ diagram is, thus, intimately related to the molar properties of a binary system.

Since the phase diagram and the thermodynamic properties of a binary system are interrelated, in principle, it should be possible to obtain some thermodynamic information about a system from its phase diagram. Conversely, it should also be possible to obtain the phase diagram from experimental thermo. 
dynamic data. Numerous binary and ternary phase diagrams have, in fact, been calculated from thermodynamic data. Until recently, most of these determinations have been done by graphical analysis of approximate representations of $\Delta \mathrm{G}_{\mathrm{m}}$. Currently, however, a great deal of work is being done in the field of computer calculation of phase diagrams from experimental data. Several articles $(45,50)$ and conference proceedings $(5,30)$ contain some of the more recent advances in this field.

Despite the large amount of interest in the calculation of phase diagrams, it is often more difficult to obtain reli: able thermodynamic data for a particular system than to obtain its phase diagram. As a consequence, systems possessing phase diagrams far outnumber those possessing extensive, reliable thermodynamic data. Phase diagrams represent a vast potential source of thermodynamic data. The thermodynamic information which can be obtained from the phase boundaries is, however, much less than can be known about a system. This is largely due to the fact that the $T-x_{i}$ diagram is only one of several projections of the thermodynamic surfaces (50). Additional information aside from the phase boundaries is often required to calculate the molar quantities of a system. In addition, the evaluation of a particular molar quantity at several compositions results in a different temperature for each $Y$ value. As a result, a solution model must be assumed in order to obtain isothermal relationships for $Y$ as a func- 
tion of composition. Many of these solution models require the tedious evaluation of large systems of simultaneous equations in order to obtain values for their parameters. Because of these problems, literature dealing with the calculation of thermodynamic properties from phase diagrams has, until recently, been somewhat limited.

Wagner (62, pp $67-75)$ has presented several approximate relations which allow the calculation of $\Delta G_{m}$ for a few specific types of binary phase boundaries. He considered expressions for solid and liquid miscibility gaps, equilibria between a liquid and an intermediate solid phase in the vicinity of a liquidus maximum, and equilibria between a pure solid and a liquid phase. Wagner also reviewed the usage of these and other phase equilibrium equations prior to 1952. Several authors $(61,67)$ have since more completely explored the calculation of $\Delta G_{m}$ for miscibility gap systems. Sundquist (58) has reviewed and compared several of the solution models used in these calculations.

As a result of the increased availability of high speed computers, work pertaining to the calculation of thermodynamic properties from phase diagrams has markedly increased over the past fifteen years. Hiskes and Tiller $(24,25,26)$ have written a series of papers on the calculation of chemical potentials from phase diagrams. Utilizing the integral form of the Gibbs-Duhem equation for binary systems, the 
authors derived the following expression for the chemical potential of the component, 1 , in the phase, $\mathrm{p}$ :

$$
\mu_{1}^{\mathrm{p}}\left(\mathrm{x}_{\mathrm{A}}, \mathrm{T}\right)=\mu_{1}^{\mathrm{p}}(\mathrm{T})+\mathrm{RT} \ln \mathrm{x}_{1}+\mathrm{f}\left(\mathrm{T}, \mathrm{x}_{1}\right)
$$

where $f\left(T, x_{1}\right)$ represents a double series expansion in $T$ and $x_{1}$. The equilibrium phase relationship, $\mu_{1}^{i}\left(T, x_{1}\right)=\mu_{1}^{j}\left(T, x_{1}^{\prime}\right)$, is implemented to obtain the coefficients of $f\left(T, x_{1}\right)$ from the phase boundaries. Rao, Hiskes, and Tiller (48) have used a similar technique to evaluate the coefficients of a parametric representation of the $\alpha$ function where

$\alpha_{12} \equiv \frac{\ln \gamma_{1}}{\left(1-x_{1}\right)^{2}}$ and $\alpha_{12}=l_{12}+a_{12} x_{2}+\frac{b_{12}}{T}+c_{12} \frac{x_{2}}{T}$ (II - 27) The parameters of Equation II-27 can be used to calculate the activity coefficient at infinite dilution:

$$
\ln \gamma_{2}^{0}=\ell_{12}+\frac{b_{12}}{T}+\frac{1}{2} a_{12}+\frac{c_{12}}{2 T}
$$

Rudman (50), in an extensive treatise on the analysis and synthesis of phase diagrams, has outlined a method of obtaining phase diagrams and thermodynamic properties for binary systems which are internally self-consistent. The article contains both equations and computer programs for the calculation of phase diagrams from thermodynamic data and for the calculation of thermodynamic properties from phase diagrams. Using power series expansions to represent $\Delta \bar{G}_{1}^{X S}$ and $\Delta \bar{G}_{2}^{X S}$, he presented several schemes for averaging the experi- 
mental phase diagram and thermodynamic properties of a system to obtain a mutually consistent set of data for the system. Rudman then applied this technique to miscibility-gap, solidliquid equilibrium with no terminal solid solubility, and finite solid solubility solid-liquid equilibrium type phase boundaries.

A recent summary of general thermodynamic relations for the calculation of thermodynamic properties from phase diagrams has been given by Chiotti, Simmons, and Kateley ( 8 ). The summary contains many useful equations and procedures for extracting thermodynamic information from phase boundary data. In addition, several empirical parametric representations of $\Delta \bar{G}_{i}^{X S}$ were evaluated using the relations developed in the paper. An equation, of particular importance to this work, for computing the difference between $\Delta \bar{G}_{i}^{X S^{\prime}}$ for phase (') and $\Delta \bar{G}_{i}^{X S^{\prime \prime}}$ for a second phase (") was developed and is given below as Equation II-29:

$$
\Delta \bar{G}_{i}^{X S^{\prime}}-\Delta \bar{G}_{i}^{X S^{\prime \prime}}=\operatorname{RT} \ln \left(\frac{X_{i}^{\prime \prime}}{x_{i}^{\prime}}\right)-\Delta G_{i}^{\circ}, " 1 \rightarrow,
$$

This equation is general and may be applied to any two-phase equilibrium boundary. The equation is valid for any number of components or phases (8) and can be derived by noting that at equilibrium the chemical potentials for a component in two phases are equal; thus:

and

$$
\bar{G}_{i}^{\prime}=\bar{G}_{i}^{\prime \prime}
$$




$$
\Delta \bar{G}_{i}^{\prime}=\Delta \bar{G}_{i}^{\prime \prime}-\Delta \bar{G}_{i}^{0, "}{ }^{\prime \prime \prime}
$$

If Equation II-31 is separated into its ideal and excess quantities, Equation II 29 is obtained upon rearrangement.

For eutectic systems with negligible or limited solid solubility, Equation II-29 simplifies to the following equation for the A-rich liquidus, $\ell$, in equilibrium with an Arich solid solution, $\alpha$, :

$$
\Delta \bar{G}_{A}^{X S}, l=\operatorname{RT} \ln \left(\frac{x_{A}^{\alpha}}{x_{A}^{\ell}}\right)-\Delta G_{A}^{\text {fus }}(T) .
$$

Since the system possesses, at most, only limited solid solubility, $\Delta \bar{G}_{\mathrm{A}}^{X S}, \alpha$ becomes very smali in comparison to $\Delta \bar{G}_{\mathrm{A}}^{X S}, l$ and can be neglected. $\Delta G_{A}^{f u s}(T)$, the free energy of fusion of component A, is calculated from data for pure A by the following relations:

$$
\begin{aligned}
& \Delta \mathrm{G}_{\mathrm{A}}^{\text {fus }}(\mathrm{T})=\Delta \mathrm{H}_{\mathrm{A}}^{\text {fus }}(\mathrm{T})-\mathrm{T}_{\Delta} \mathrm{S}_{\mathrm{A}}^{\text {fus }}(\mathrm{T}) \\
& \Delta \mathrm{H}_{\mathrm{A}}^{\text {fus }}(\mathrm{T})=\Delta \mathrm{H}_{\mathrm{T}_{\mathrm{m}}}^{\mathrm{O}}+\int_{\mathrm{T}_{\mathrm{m}}}^{\mathrm{T}} \Delta \mathrm{C}_{\mathrm{p}} \mathrm{dT} \\
& \Delta \mathrm{S}_{\mathrm{A}}^{\text {fus }}(\mathrm{T})=\Delta \mathrm{S}_{\mathrm{T}_{\mathrm{m}}}^{\mathrm{o}}+\int_{\mathrm{T}_{\mathrm{m}}}^{\mathrm{T}} \frac{\Delta \mathrm{C}_{\mathrm{p}}}{\mathrm{T}} \mathrm{dT}
\end{aligned}
$$

$\Delta \mathrm{H}_{\mathrm{T}_{\mathrm{m}}}^{\mathrm{o}}$ and $\Delta \mathrm{S}_{\mathrm{T}_{\mathrm{m}}}^{\mathrm{o}}$ are the standard enthalpy and entropy of fusion at the melting temperature $\mathrm{I}_{\mathrm{m}}$. Simmons (57) has used Equation II-32 and others given in this summary to evaluate various empirical equations for the thermodynamic description of salt and metal systems. 
Petot-Ervas, Petot, Desré, and Bonnier (47) have written a paper of central importance to this investigation on the calculation of interaction parameters from phase boundary. data. The authors have derived the following expression which may. be used to estimate self-interaction parameters from the slopes of liquidus and solidus data:

$$
\begin{aligned}
& \left.-\varepsilon_{2}^{2},{ }^{\prime}+\rho^{02} \varepsilon_{2}^{2}, "=\left(\frac{\mathrm{dT}}{\mathrm{dx}}\right)_{2}^{\prime}\right)_{x_{2}^{\prime}=0}\left\{\frac{-2 p^{0}}{\mathrm{~T}_{\mathrm{f}_{1}}}\left(1+\frac{\Delta \mathrm{H}_{f_{1}}^{\circ}}{2 \mathrm{RT}_{\mathrm{f}_{1}}}\right)\right.
\end{aligned}
$$

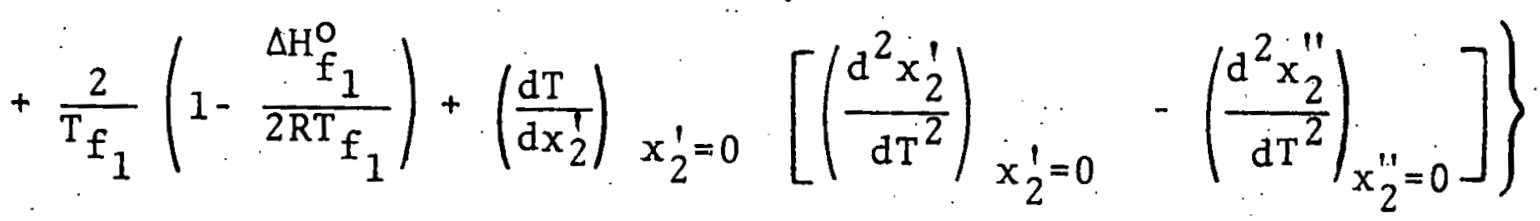

The self-interaction parameter for the liquid solution is

$$
\varepsilon_{2}^{2},^{\prime}=\left(\frac{\partial \ln \gamma_{2}^{\prime}}{\partial x_{2}^{\prime}}\right) x_{2}^{\prime}=0
$$

and

$$
\varepsilon_{2}^{2}, "=\left(\frac{\partial \ln \gamma_{2}^{\prime \prime}}{\partial x_{2}^{\prime \prime}}\right) x_{2}^{\prime \prime}=0
$$

is the self-interaction parameter fur the solid solution. The superscripts (') and (') represent the liquid and solid phases respectively and 1 and 2 signify the solvent and the solute. $\rho^{\circ}$ is defined in the following manner:

$$
\rho^{0}=\left(\frac{x_{2}^{\prime \prime}}{x_{2}^{\prime}}\right)_{x_{2}^{\prime}=x_{2}^{\prime \prime}=0}=\left(\frac{d x_{2}^{\prime \prime}}{d x_{2}^{\prime}}\right)_{x_{2}^{\prime}=x_{2}^{\prime \prime}=0}
$$


$\Delta \mathrm{H}_{\mathrm{f}_{1}}^{\circ}$ is the heat of fusion of pure component 1 and $\dot{\mathrm{T}}_{f_{1}}$ is the pure component melting temperaturè. Although Petot-Ervas et a1. have derived the equation by some other procedure, Equation II-36 can be obtained by differentiating Equation II-29 twice with respect to $T$ and taking the limit of the resulting expression as $x_{1}^{\prime} \rightarrow 1$ (or $\left.\ddot{x}_{2}^{\prime} \rightarrow 0\right)$. If $\Delta \bar{S}_{1}^{X S}$. and $\Delta \bar{H}_{1}^{X S}$ are assumed to be independent of temperature, and Raoult's law is assumed to hold, the limiting expression can be simplified to Equation II-36.

Petot-Ervas et a1. have used Equation II-36 to. calculate the solid-solution self-interaction parameter, $\varepsilon_{2}^{2}$,", for the $\mathrm{Cu}-\mathrm{Zn}, \mathrm{Ag}-\mathrm{Au}$, and $\mathrm{Cu}-\mathrm{Ag}$ systems. Utilizing a third degree power series polynomial to obtain the derivatives in Equation I - 36 and thermodynamic data from the literature to obtain $\varepsilon_{2}^{2}$, , the authors calculated values for $\varepsilon_{2}^{2}, "$ and compared them with values obtained from thermodynamic measurements. Except for the Ag-Au system, the values were not in very good agreement. More recently, Martin-Garin, Petot-Ervas, Petot, and Bonnier (43) have developed an equation for estimating binary and ternary self-interaction parameters from monotectic type phase diagrams. Since their equation applies only to monotectic diagrams, it will not be further considered in this work.

Chiotti (7) has also derived an equation for the calculation of self-interaction parameters from phase diagrams which 
can be shown to be related to Equation II-36. Using the notation of Petot-Ervas et al., the equation is given by:

$$
\begin{aligned}
-\varepsilon_{2}^{2},+\rho^{\circ 2} \varepsilon_{2}^{2}, "=\left(1-\rho^{\circ 2}\right)=\left(1-\rho^{\circ}\right)^{2} \frac{{ }^{2 R T_{f_{1}}}}{\Delta S_{f_{1}}^{0}} \\
\left.+\frac{\Delta S_{f_{1}}^{\circ}}{}+\frac{d^{2} T}{R_{f_{1}}}\right)_{x_{1}^{\prime} \rightarrow 1}+\left(\frac{d^{2} x_{1}^{\prime \prime}}{d x_{1}^{\prime 2}}\right)_{x_{1}^{\prime} \rightarrow 1}
\end{aligned}
$$

Equation II-38 can be derived in a manner similar to Equation II-36 by differentiating. Equation II-29 twice with respect to $x_{1}^{\prime}$ rather than with respect to $T$ as in Equation II-36. Equations II-36 and II-38 are equivalent expressions since Equation II-38 can be converted into Equation II-36 by a change of variable. If limited solid solubility is assumed, Equation II-38 simplifies to the previously discussed (see page 4 Equation.I-10). Equation I-10 can be used to calculate the liquid self-interaction parameter, $\varepsilon_{2}^{2}, '$. If negligible solid solubility is assumed, Equation II-38 can be further simplified to the relation:

$$
\varepsilon_{2}^{2}, '=\frac{2 R}{\Delta S_{f_{1}}^{0}}-1-\frac{\Delta S_{f_{1}}^{0}}{R T_{f_{1}}}\left(\frac{d^{2} T}{d x_{1}^{\prime 2}}\right)_{x_{1}^{\prime \rightarrow 1}}
$$

The derivation of Equations II-38, I-10, and II-29 are given in Appendix A. Equations I-10 and II-39 will be used to calculate the self-interaction parameters in this investigation. 
III. METHODS OF DETERMINING SELF-INTERACTION PARAMETERS FROM EXPERIMENTAL DATA

A. Determination from Activity Coefficient Measurements

At present, experimental values for the self-interaction parameters are obtained almost exclusively by the analysis of experimentally determined activity coefficient data. Depending on the particular solute or solutes in question, these activity coefficient data can be obtained by a variety of experimental techniques. Galvanic cells, vapor pressure measurements, and various equilibrium distribution techniques are among the most frequently used methods. Regardless of the technique, precise dilute solution data are necessary if the interaction parameters--particularly the second order parameters--are to be determined with any degree of certainty. Because the galvanic cell technique is capable of a high degree of precision, it is currently the most popular method of obtaining data for the determination of interaction parameters. Vapor pressure measurements are also frequently used for solutes with a measurable vapor pressure in some temperature range of interest.

Values for the interaction parameters can be calculated from these thermodynamic measurements by several analytical methods. Perhaps the most direct way of determining the parameters is by graphical analysis of various isothermal $\ln \gamma_{i}$ versus composition graphs. Gluck and Pehlke (17) have out- 
lined the following graphical methods for determining interaction parameters. The self-interaction parameter, $\varepsilon_{i}^{i}$, can be determined by simply plotting $\ln \gamma_{i}$ versus $x_{i}$, drawing a smooth curve through the data points, and obtaining the graphical slope at $x_{i}=0$. This slope is the self-interaction parameter. The ternary parameter, $\varepsilon_{i}^{j}$, of the $1-i-j$ system may also be determined graphically. $\ln _{i}$ versus $x_{j}$ curves are obtained for various constant values of $x_{i}$ and the slope of each of these curves is determined at $x_{j}=0$. A plot of these

$$
\omega_{i}^{j} \equiv\left(\frac{\partial \ln \gamma_{i}}{\partial x_{j}}\right)_{\substack{x_{i}=\text { constant } \\ x_{j}=0}}
$$

slopes versus $x_{i}$ is then drawn. The slope of this $\omega_{i}^{j}$ versus $x_{i}$ curve at $x_{i}=0$ is the ternary parameter,

$$
\varepsilon_{i}^{j}=\left(\frac{\partial \ln \gamma_{i}}{\partial x_{j}}\right)_{\substack{x_{i}=0 \\ x_{j}=0}}
$$

In addition to these graphical procedures, several numerical techniques are also available for the calculation of binary and ternary interaction parameters. If the thermodynamic data are of sufficient accuracy to permit the calculation of both the first and second order parameters, the so-called "statistical method" (19) may be used to simultaneously calculate $\varepsilon_{i}^{i}$ and $\rho_{i}^{i}$. The interaction parameter representation for a binary system may be expressed as 


$$
\ln \gamma_{i}=\ln \gamma_{i}^{o}+\varepsilon_{i}^{i} x_{i}+\rho_{i}^{i} x_{i}^{2}
$$

If Equation III- 1 is rearranged to the following form:

$$
Y_{1-i}=\left(\ln \gamma_{i}-\ln \gamma_{i}^{o}\right) / x_{i}=\varepsilon_{i}^{i}+\rho_{i}^{i} x_{i}
$$

and the experimental activity coefficient data are submitted to a linear least squares treatment using Equation III-2, the self-interaction parameter, $\varepsilon_{i}^{i}$, and the binary second order parameter, $\rho_{i}^{i}$, can be obtained as the respective intercept and slope of Equation III-2.

The ternary interaction parameters can be obtained by a similar but slightly more involved procedure. The ternary interaction parameter representation given by Equation II- 8 is rearranged to the following form:

$Y_{1-i-j}=\frac{\left(\ln \gamma_{i}-\ln \gamma_{i}^{o}\right)-\varepsilon_{i}^{i} x_{i}-\rho_{i}^{i} x_{i}^{2}}{x_{j}}-\rho_{i}^{i, j}=\varepsilon_{i}^{j}+\rho_{i}^{j} x j$

After determining $\varepsilon_{i}^{i}$ and $\rho_{i}^{i}$ by the method described in the previous paragraph, various values for $\rho_{i}^{i, j}$ are arbitrarily chosen and values for $\varepsilon_{i}^{j}$ and $\rho_{i}^{j}$ are calculated by linear least squares regression. The set of values for $\rho_{i}^{i, j}, \varepsilon_{i}^{j}$, and $\rho_{i}^{j}$ that yield the smallest standard deviation for $Y_{1-i-j}$ are taken as the best values for the parametcrs.

Pelton and Thompson (45) have criticized these statistical procedures for determining interaction parameters. The authors point out that the least squares method yields parameters which are highly correlated and are, thus, not equivalent 
to the partial derivatives of the Taylor's series. The authors contend that any method for obtaining the interaction parameters from $1 \mathrm{n} \gamma_{i}$ data should involve determining the various partial derivatives at $x_{i}=0$ rather than using Equations II- 8 and III-1 to do statistical curve fitting.

In addition to the statistical method, Hadrys, Frohberg, and Elliott (19) have also outlined a rather specialized technique for ternary systems which utilizes activity data measured at constant chemical potential of one of the solutes. The method utilizes polynomial descriptions of various $\ln _{i}$ versus $x_{i}$ plots at constant $\mu_{j}$ to obtain constant chemical potential interaction parameters. These parameters are then converted to the traditional interaction parameters defined by Equation II-I by means of various conversion equations. Since this technique is somewhat limited in its application and cannot be applied to binary systems, it will not be further considered.

Empirical representations of $\Delta \bar{G}_{i}^{X S}=f\left(T, x_{i}\right)$ can also be used as a means of determining self-interaction parameters from experimental thermodynamic data. A number of these equations exist (see page 6) and, depending on their ability to accurately represent the particular system in question, many of these equations could potentially be used to numerically calculate interaction parameters. Chiotti, Simmons, and Kateley (8) have found the following equation to be fairly 
successful in representing a number of binary systems summarized by Hultgren, Orṛ, Anderson, and Kelley (28):

$$
\Delta \bar{G}_{1}^{X S}=\left(1-x_{1}\right)^{2}\left[(\alpha+\beta T)+\left(\alpha^{\prime}+\beta^{\prime} T\right) x_{1}+\left(\alpha^{\prime \prime}+\beta^{\prime \prime} T\right) x_{1}^{2}\right]
$$

$\alpha, \beta, \alpha^{\prime}, \beta^{\prime}, \alpha^{\prime \prime}$, and $\beta^{\prime \prime}$, are determined from the experimental data by the Gauss Method (44, pp. 557.563) of solving over= determined equations. An expression for the self-interaction parameter of component 1 may be obtained by differentiating. Equation III- 4 with respect of $x_{1}$ at constant $T$, and taking the 1 imit as $x_{1} \rightarrow 0$ :

$$
\varepsilon_{1}^{1} \equiv\left(\frac{\partial \ln \gamma_{1}}{\partial x_{1}}\right)_{x_{1} \rightarrow 0}=\frac{\alpha^{\prime}-2 \alpha}{R T}+\frac{\beta^{\prime}-2 \beta}{R}
$$

An equation for the self-interaction parameter of component 2 can be obtained by utilizing the following expression to relate Equation III -4 , to $\varepsilon_{2}^{2}$ :

$$
\lim _{x_{1} \rightarrow 1} \frac{\Delta \bar{G}_{1}^{X S}}{\left(1-x_{1}\right)^{2}}=-\frac{1}{2} \operatorname{RT} \varepsilon_{2}^{2}
$$

This expression has been dcrived by Chiotti (6). Taking the limit of Equation III-4 as $x_{1} \rightarrow 1$ and substituting the expression yields the following equation for the self-interaction parameter of component 2 :

$$
\varepsilon_{2}^{2}=-\frac{2}{R}\left[\frac{\left(\alpha+\alpha^{\prime}+\alpha^{\prime \prime}\right)}{\mathrm{T}}+\left(\beta+\beta^{\prime}+\beta^{\prime \prime}\right)\right]
$$

Similar procedures could also be applied to other analytical representations to obtain expressions for the numerical calcu- 
lation of interaction parameters. Equations III-5 and III-6 were used to calculate the self-interaction parameters of several systems for comparison with the values obtained by Equation I-10.

B. Determination from Thermodynamic Data Obtained from Binary Phase Diagrams

In addition to the use of experimental activity coefficient data, it should also be possible to determine the selfinteraction parameter by using $\Delta \overline{\mathrm{G}}_{\dot{I}}^{X S}$ data obtained from binary phase diagrams. For binary eutectic systems of limited or negligible solid solubility, Equation II-32 can be used to generate $\Delta \bar{G}_{i}^{X S}$ values for the liquid phase from thermodynamic information for the pure component and the liquidus and solidus phase boundaries. Since each of the $\Delta \dot{G}_{\dot{i}}^{X S}$ values obtained from Equation II-32 are at a different temperature and composition, some type of analytical equation which is a function of both $T$ and $x_{i}$ must be used to correlate the consecutive data points. If a suitable parametric expressiun can be obtained which will adequately describe the thermodynamic data, an equation for the calculation of self-interaction parameters may be obtained by differentiating this parametric expression with respect to $x_{i}$ and taking the limit of the resultant expression as $x_{i} \rightarrow 0$.

During the course of this investigation, several empirical representations of $\Delta \bar{G}_{i}^{X S}=f\left(T, x_{i}\right)$ were examined for their potential usefulness in calculating self-interaction parameters. 
The following empirical equations were used in conjunction with Equation II-32 to obtain expressions which could be used to calculate the self-interaction parameters:

$$
\begin{aligned}
& \Delta \bar{G}_{A}^{X S}=\left[a+b x_{A}^{n}\right]\left(1-\dot{x}_{A}\right)^{2} \\
& \Delta \bar{G}_{B}^{X S}=\left[a+b x_{A}^{n-1}\left(x_{A}-\frac{n}{n+1}\right)\right] x_{A}^{2} \\
& \Delta \bar{G}_{A}^{X S}=\left[a+b x_{A}+c x_{A}^{n}\right]\left(1-x_{A}\right)^{2} \\
& \Delta \bar{G}_{B}^{X S}=\left[a+\left(x_{A}-\frac{1}{2}\right) b+c x_{A}^{n-1}\left(x_{A}-\frac{n}{n+1}\right)\right] x_{A}^{2}
\end{aligned}
$$

$a=\alpha+\beta T, b=\alpha^{\prime}+\beta^{\prime} T$, and $c$ can be either $c=\alpha^{\prime \prime}+\beta^{\prime \prime} T$ or $c=\alpha^{\prime \prime}, \alpha, \beta, \alpha^{\prime}, \beta^{\prime}, \alpha^{\prime \prime}, \beta^{\prime \prime}$, and $n$ are constants which are to be determined from the phase diagram by the use of Equation II-32. Equation III-7B can be derived from Equation III-7A using the Gibbs-Duhem equation. Equation III-8B can likewise be obtained from Equation III-8A.

Using Equations III-7A and III-7B as an example, the following procedure was used to calculate the self-interaction parameters. Equation II-32 was used to obtain $k$ values for $\Delta \bar{G}_{A}^{X S}$ from the A-rich liquidus and solidus. Substituting these values into Equation III-7A created a set of $k$ observation equations. After being rewritten in terms of the B-rich liquidus (replacing the subscript $A$ in Fquation II-32 with subscript B), Equation II-32 was used to obtain L values of $\Delta \bar{G}_{B}^{X S}$ from the $B-r i c h$ liquidus. These values were substituted into Equation III-7B to yield L observation equations for the 
B-rich liquidus. The k equations for the A-rich liquidus were combined with the L equations for the B-rich liquidus to yield a set of $m=k+L$ observation equations. The value of $n$ in Equations III-7A and III-7B.was fixed at some arbitrary value. The solution of the resultant set of m equations and 4 unknowns was accomplished by using the Gauss Method (44, pp. 557-563) for overdetermined equations. By a series of multiplication and addition steps, the Gauss Method converted the $\mathrm{m}$ by 4 set of equations into a 4 by 4 set which can be solved by standard matrix methods.

The value of $n$ was varied from $n=1$ to $n=6$ by increments of 0.1 and the constants along with the standard deviation were calculated for each value of $n$. The values of $n, \alpha, \beta$, $\alpha^{\prime}$ and $\beta^{\prime}$ ' which gave the lowest standard deviation were taken as the best values for the expression.

Once the coefficients of the equation had been determined, the self-interaction parameters were calculated using the following expressions:

$$
\begin{aligned}
& \varepsilon_{A}^{A}=-\frac{2}{R T}[\alpha+\beta T] \\
& \varepsilon_{B}^{B}=-\frac{2}{R T}\left[(\alpha+\beta T)+\left(\alpha^{\prime}+\beta^{\prime} T\right)\right]
\end{aligned}
$$

Fquations III-9 and III-9B were dertved by differentiating the parametric equations with respect to $x_{i}$ and taking the limit as $x_{i} \rightarrow 0$. The corresponding expressions for Equations III-8A and III-8B are: 


$$
\begin{aligned}
\varepsilon_{A}^{A} & =-\frac{\left[2(\alpha+\beta T)-\left(\alpha^{\prime}+\beta^{\prime} T\right)\right]}{R T} \\
-\varepsilon_{B}^{B} & =-\frac{2}{R T}\left[(\alpha+\beta T)+\left(\alpha^{\prime}+\beta^{\prime} T\right)+\left(\alpha^{\prime \prime}+\beta^{\prime \prime} T\right)\right] .
\end{aligned}
$$

As a preliminary test, values for the self-interaction parameters of five systems were determined using the empirical representations defined by Equations III-7A and III-8A. In all of the determinations, the expressions failed to give reasonable values for the self-interaction parameters. The values ranged from $\varepsilon_{A}^{A}=+10$ to $\varepsilon_{A}^{A^{-}}=+500$ for the five systems determined. It is not known at this time why this method yielded such poor results. The problem may be a consequence of the approximate nature of the expressions used or it may be due to the particular numerical methods used to determine the constants of the expressions. In any event, further work would be required to ascertain the cause of these poor results.

\section{Determination Directly from Binary Phase Diagrams}

An alternate method is available for the calculation of self-interaction parameters from binary phase diagrams. For binary liquidus-solidus systems possessing limited or negligible solid solubility, the previously discussed Equations I-10 and II-39 (see pages 4 and 30) can be used to determine the self-interaction parameter directly from the liquidus and solidus phase boundaries. Equation I-10 utilizes various first and second derivatives from the A-rich phase boundaries 
to calculate $\varepsilon_{B}^{B}$, the self-interaction parameter for the $B$ component of the A-B binary system. Only two curves must be considered in order to obtain the derivatives in Equation I-10. Evaluation of the first and second derivatives of the A-rich liquidus curve at $x_{A}^{\prime}=1$ gives

$$
\left(\frac{\mathrm{d} T}{d x_{A}^{T}}\right)_{x_{A^{\prime}+1} \text { and }}\left(\frac{d^{2} T}{d x_{A}^{\prime 2}}\right)_{x_{A}^{\prime} \rightarrow 1} \text {. }
$$

The quantities

$$
\left(\frac{\mathrm{d} \ln \frac{x_{A}^{\prime}}{x_{A}^{\prime \prime}}}{d x_{A}^{\prime}}\right)_{x_{A \rightarrow 1}^{\prime}} \text { and } \quad\left(\frac{d^{2} \ln \frac{x_{A}^{\prime}}{x_{A}^{\prime \prime}}}{d x_{A}^{\prime 2}}\right)_{x_{A}^{\prime} \rightarrow 1}
$$

are evaluated from a plot. of $\ln \left(x_{A}^{\prime} / x_{A}^{\prime \prime}\right)$ versus $x_{A}^{\prime}$ where $x_{A}^{\prime \prime}$ represents the isothermal tie-line value of the solidus for some corresponding $x_{A}^{\prime}$ liquidus value. Equation II-39, the equation for cases of negligible solid solubility, requires the determination of only

$$
\left(\frac{d^{2} T}{d x_{A}^{\prime 2}}\right)_{x_{A}^{\prime}+1}
$$

A number of numerical analysis techniques are available for calculating the derivatives used in Equation I-10. Various polynomial curve fitting methods and direct numerical differentiation techniques are given in standard texts $(32,63)$ on . numerical analysis. Some caution should be used when applying these methods to experimental data. Many. of these techniques 
are highly sensitive to random errors in the original data and the direct application of these methods to imprecise experimental data can, in some cases, yield less than satisfactory results. Differentiation is an error amplifying process and special care must be taken in the utilization of these methods to minimize the effects of random errors. For example, curves which are to be submitted to direct numerical diffcrentiation techniques often must be smoothed to obtain reasonable values for the derivatives. Care must also be taken when utilizing the polynomial curve fitting methods to insure that the analytical expression is fitting the functional behavior of the data and not the random scatter of the data points. If carefully monitored, however, many of these methods can be suc: cessfully used to obtain derivatives from numerical data.

The major emphasis of this investigation has been to examine several numerical techniques for their usefulness as a means of calculating the derivatives required by Equations I-10 and II-39. Both a method which utilizes least squares curve fitting to obtain an analytical expression for subsequent differentiation and various methods involving direct numerical differentiation were examined. Least squares curve fitting by means of a simple power series polynomial was utilized as one method of obtaining derivatives for Equations I-10 and II-39. Several direct differentiation methods were also examined. One of these, the graphical derivative method, 
was chosen as a second method for obtaining the derivatives required by the equations. In the following discussions; some general features of these methods will be considered and the particular procedures which were used to calculate the selfinteraction parameters will be given.

One method of determining the derivatives required by Equations I-10 and II-39 consists of least squares curve fitting the phase boundary data with a suitable analytical expression and then subsequently differentiating and evaluating the expression at $x_{A}^{\prime}=1.0$. Since no theoretical equation for the functional dependence of the phase boundary data is known, some type of polynomial or other analytical equation must be used to approximate the functional dependence implied by the tabular data. Any of a vast number of polynomials could potentially be used to approximate the data. The Legendre, Fourier, Chebyshev, Taylor's series, and power series polynomials are a few of the more frequently used curve fitting representations. Selecting the best possible polynomial to approximate the data would be difficult as an almost infinite number of possibilities exist. Because of their familiarity and simplicity, the power series polynomials were used in this study to fit the phase boundary data. The power series polynomial representation is given by the following expression:

$$
Y=\sum_{n=0}^{N} \cdot c_{n} x^{n}=c_{0} x+c_{2} x^{2}+c_{3} x^{3}+\ldots+c_{N} x^{N}
$$


where $\mathrm{N}$ is the polynomial degree and $c_{0}, c_{1}, \ldots, c_{N}$ are the constants to be determined from the tabular data.

After specifying a particular value for $N$, the coefficients of Equation III-11 may be obtained by using the method of least squares (46, pp. 408-417). Letting i represent some particular $(x, y)$ data point and letting $r_{i}$, the residual, represent the difference between the actual $Y$ value and the $Y$ value calculated from the polynomial, the method of least squares yields coefficients such that the value of $R$ in the following expression is a minimum:

$$
R=\sum_{i=1}^{m} r_{i}^{2}
$$

$m$ is the number of data points. The method of least squares thus, yields the particular polynomial which will minimize the sum of the squares of the differences between the experimental data and the analytical curve. The least squares method involves a number of tedious calculations and because of this, computer programs are generally used to perform the curve fitting. Since the method of least squares is given in most texts $(32,46,63)$ on numerical analysis, the details of the method will not be discussed further. A program given by Pennington (46, p. 413) was used in this investigation to perform the curve fitting. The program utilizes Gausian elimination to solve the set of simultaneous equations created by the least squares treatment.

The selection of an appropriate degree of power series 
polynomial to curve fit the experimental data depends largely on how the resultant analytical expression will be used. Using the least squares method, any polynomial degree from $\mathrm{N}=0$ to $N=m$, where $m$ is the number of data points, may be used to fit the data. A value of $N=0$ would, of course, fit the data points with a horizontal straight line. On the other hand, a polynomial of degree $\mathrm{N}=\mathrm{m}$ would pass through all of the data points exactly but in doing so, would oscillate violently between the points. A polynomial of somewhat lesser degree would not oscillate as violently but the curve would still possess a certain amount of ripple. If the degree were to be further decreased, the analytical curve would become smoother but in the process, the error in the fit would increase. Some compromise between an exact fit and a straight line is usually taken to be the "best fit". For interpolation and numerical integration, several rules of thumb are sometimes given ( $46, \mathrm{p}$. 406) for selecting an appropriate value of $N$. One rule states that the degree should be $1 / 2$ to $3 / 4$ of the number of data points while another states that the polymial degree should be no larger than the square root of the number of data points. When the analytical function is to be used to obtain derivatives, a polynomial of low degree should be used in order to reduce the amount of unwarranted ripple in the curve and to obtain the maximum amount of smoothing. The choice of degree, however, is usually somewhat arbitrary and is highly dependent 
on the situation in question.

When power series polynomials are to be utilized for obtaining derivatives, certain requirements must be placed on the polynomial. If the first and second derivatives are to be obtained from the polynomial, the analytical curve must not only reproduce the original data reasonably well, it must also match the slope and curvature implied by the experimental data as we11. Perhaps the most direct method for assessing whether a particular polynomial accomplishes this is to actually plot the analytical curve along with the data points and visually determine whether or not the analytical curve is fitting the functional form of the data. The analytical curve should flow smoothly through the data points without ripples and should reproduce the curvature throughout the region where the derivatives are to be evaluated. Selecting the polynomial which best fits the data is largely a matter of distinguishing between the random errors in the experimental data and the inherent functional properties displayed by the data.

Values for the derivatives--especially the second derivatives--are somewhat more difficult to obtain at the endpoints than in the central region of an interval. Because no functional information is available beyond the endpoints, the power series curve fitting functions sometimes do not adhere we 11 to the curvature displayed by the experimental data near the end of the interval. Although the analytical curve may pass 
through the endpoint, the fitting function may rapidly curve upward or downward or take on some other type of curvature uncharacteristic of the experimental data points. Since the second derivative represents the curvature, this type of behavior can have a drastic effect on the calculated values of the second derivative. In many cases, this problem can be alleviated by using artificial data points beyond the endpoint to force the fitting function to maintain a curvature characteristic of the experimental data. These artificial data points can be obtained by drawing a smooth curve through the data points and extrapolating the curve past $x_{A}^{\prime}=1$, taking care in the process to continue the change in curvature indicated by the data points prior to $x_{A}^{\prime}=1$. Admittedly, some error may be introduced into the derivatives through the use of this graphical procedure but these errors are almost certain to be less than the errors caused by allowing the function to be uncontrolled beyond the endpoint. In addition to this extrapolation procedure, the utilization of more data points or the weighting of the existing points near the end of the interval can also be of help in insuring that the polynomial accurately represents the functional form of the data at the endpoint. Regardless of the procedures used, it is essential that the analytical curve match as closely as possible the functional properties displayed by the data in the region around the point $x_{A}^{\prime}=1$. 
The following procedure was used to calculate the first and second derivatives of the phase boundary data required for Equations I-10 and II-39. Data points were read from the phase diagrams of Hansen and Anderko (20) at intervals of 0.05 atomic fraction, starting with the eutectic, by using a grid.with graduations at every 0.01 atomic fraction. The data points were plotted and a smooth curve was then drawn through the points. The curve was extrapolated to $x_{A}^{\prime}=1.05$ taking care to continue the change in the curvature indicated by the data points. Two or three data points were read from the extrapolated curve and added to the data set. The data points were then fitted with a first, second, third, and fourth degree power series polynomial. Each of these curves were computer plotted along with the data points to determine which of the four curves most closely. fit the experimental data points near and at $x_{A}^{\prime}=1.0$. The curve which adhered the best to the functional dependence displayed by the data points was chosen as the polynomial with the "best fit". If none of the curves appeared to give a satisfactory fit at $x_{A}^{\prime}=1.0$, the data points in the region of $x_{A}^{\prime}=1.0$ were given extra weight and the process was repeated. Once a suitable fit had been obtained, the values for the derivatives were calculated from the differentiated form of the polynomial and the self-interaction parameter was then calculated using either Equation I-10 or II-39. This curve fitting procedure is somewhat subject to the 
bias of the observer since it is dependent on the observer to visually judge which curve has given the "best fit". Although in most cases this decision is not difficult, occasionally the observer is required to make a rather arbitrary judgement as to which curve best fits the data. For example, the second, third, and fourth degree polynomials for the Pb-rich liquidus of the $\mathrm{Cd}-\mathrm{Pb}$ system all seem to fit the data equally well yet their second derivatives are $+957,+589$, and -195 respectively. The resultant interaction parameters are $-2.4,-1.8$, and -0.6 . A plot of each of these polynomials is given in Figure 1. Sometimes the use of a large number of data points can be of help in selecting the best curve. Also, consideration should be given as to whether or not the polynomial is following the extrapolated curvature implied by the experimental data. In this investigation, when no definitive decision could be made, the polynomial of lowest degree was chosen.

In order to obtain an estimate of the amount of uncertainty in the self-interaction parameter determined by power se: ries curve fitting, a test curve was created using a fifth degree power series polynomial. The polynomial curve was computer plotted to the same scale as the diagrams of Hansen and Anderko (20) and was similar in shape and dimensions to a common 1 iquidus curve. The scale was such that the data points could be read to only about three significant figures of accuracy. Since the test curve was obtained from an analytical function, the first and second derivatives were known exactly. 

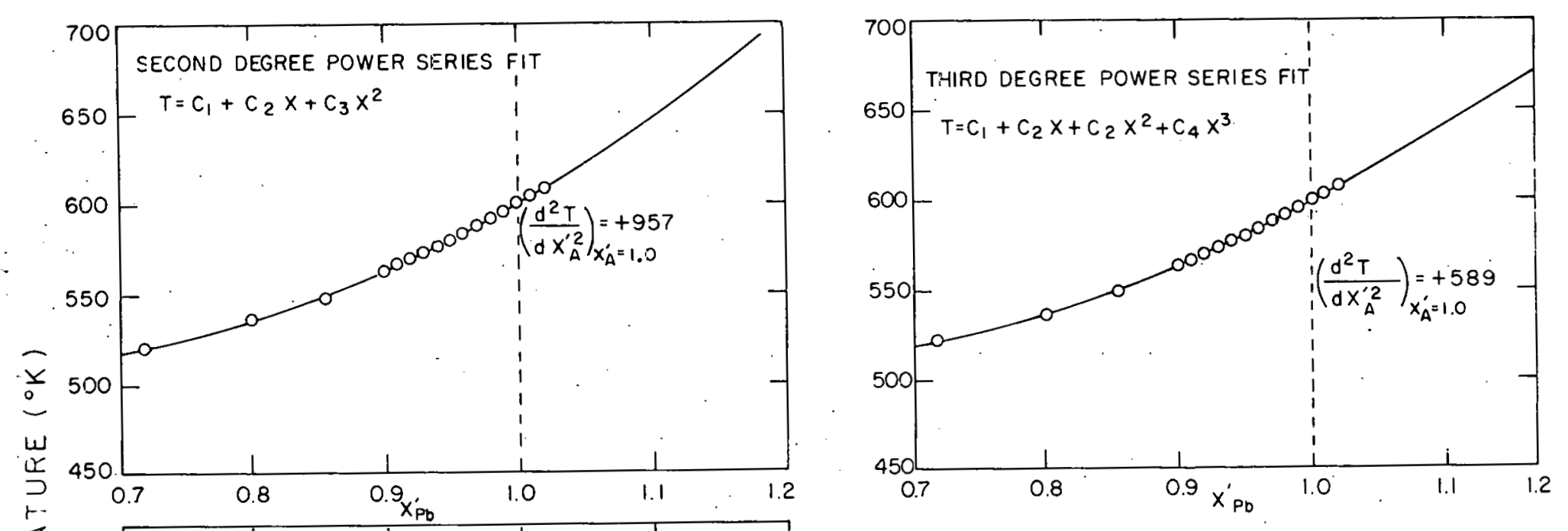

Figure 1. Cd-Pb system, Pb-rich liquidus. Plots of second, third, and fourth degree power, series polynomials. All of the polynomials give a reasonable fit. 
Data points. were read from the test curve and submitted to the previously described curve fitting procedure. The results of the test indicated that the se1f-interaction parameter could be calculated to no better than about \pm 1.5 from the diagram. Another way of determining the derivatives of the phase boundary data is through the use of direct numerical differentiation formulas. These differentiation formulas can be used to calculate the derivatives directly from the tabular data without recourse to a least squares treatment of the data. A number of central difference and "interpolation polynomial" formulas are available and many others could be derived. The central difference formulas utilize data points on both sides of $f^{\prime}\left(x_{0}\right), x_{0}$ being the point where the derivative is to be evaluated, while the backward and the forward interpolation polynomials utilize information on only one side of $f^{\prime}\left(x_{0}\right)$. Both the central difference and the interpolation formulas make use of the successive first, second, third, etc. differences of the tabular $f(x)$ values to create the coefficients of the formulas. In addition to these formulas, the method of undetermined coefficierts. (15, pp. 95-99) can be used to derive formulas which utilize the $f(x)$ values directly without recourse to the differencing operations.

During the course of this investigation, three of these methods were briefly examined to determine their potential for calculating the derivatives of the phase boundaries. Formulas utilizing the last five data points, including the endpoint, 
were derived for the first. and second derivatives using the method of undetermined coefficients outlined by Gerald (15, pp. 95-99). The formulas are given below:

$$
\begin{aligned}
& f_{0}^{\prime}=\frac{3 f_{-4}-16 f_{-3}+36 f_{-2}-48 f_{-1}+25 f_{0}}{12 \Delta x} \\
& f_{0}^{\prime \prime}=\frac{11 f_{-4}-56 f_{-3}+114 f_{-2}-104 f_{-1}+35 f_{0}}{12 \Delta x^{2}}
\end{aligned}
$$

where $f_{0}$ is the tabular value of the function at $x_{A}^{\prime}=1.0$ and $f_{-i}$ represents the ith data point preceding $f_{0}$. The data points must be equally spaced. The increment, $\Delta x$, is given by $\Delta x=$ $x_{0}-x_{-1}$. An interpolation polynomial given by Whittaker and Robinson (64, pp. 62-63) was also examined. The equations for the first and second derivatives are obtained by differentiating the interpolation polynomial. The particular polynomial used in this case is known as the Gregory-Newton backward difference formula. The derivative formulas are given below:

$$
\begin{aligned}
& f_{0}^{\prime}=\frac{1}{\Delta x}\left[\Delta f_{-1}+\frac{1}{2} \Delta^{2} f_{-2}+\frac{1}{3} \Delta^{3} f_{-3}+\ldots\right] \\
& f_{0}^{\prime \prime}=\frac{1}{\Delta x^{2}}\left[\Delta^{2} f_{-2}+\Delta^{3} f_{-3}+\frac{11}{12} \Delta^{4} f_{-4}+\ldots\right]
\end{aligned}
$$

where $\Delta f_{i}=f_{i+1}=f_{i}$ or in general, $\Delta \dot{i}_{i}$ represents the $i$ th backward difference.

Lastly, a graphical method utilizing the simple central difference formula:

$$
f_{0}^{\prime}=\frac{f_{-1}-f_{1}}{\Delta x}
$$


was examined. The first derivative was calculated at equal increments over the entire range of the curve. A plot of $f^{\prime}$ versus $x$ was then made and a smooth curve was carefully drawn through the data points and extrapolated to $x_{A}^{\prime}=1.0$. The first derivative was obtained by noting the value at $x_{A}^{\prime}=1.0$. Data points were then read from the smoothed curve and used to calculate the second derivatives over the entire range by Equation III-16. 'A smooth curve was drawn through the f' versus $x_{A}^{\prime}$ plot and extrapolated to yield the second derivative at $x_{A}^{\prime}=1.0$.

In order to facilitate the examination of these methods, four test curves, similar in shape and dimension to the 1iquidus curves, were constructed using various third, fourth and fifth degree power series polynomials. In an attempt to duplicate the conditions which would be encountered with the actual phase boundary data, the curves were computer plotted to the same scale as the phase diagrams of Hansen and Anderko (20). The four curves used in the examination are given in Figure 2 .

The coefficients of the various power series polynomials used to create the curves in Figure 2 are given in Table 2 . Since these curves were obtained from known analytical functions, the data pnints and the first and second derivativos at $x_{A}^{\prime}=1.0$ were known exactly. Values were read from the curves at $x_{A}^{\prime}=0.05$ increments and these values were then used to exam- 


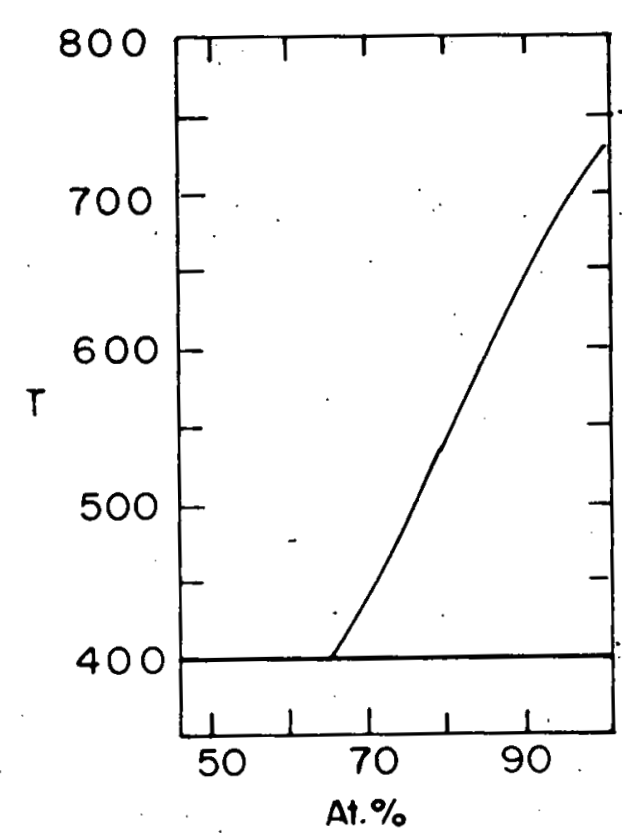

TEST DIAGRAM\# 1

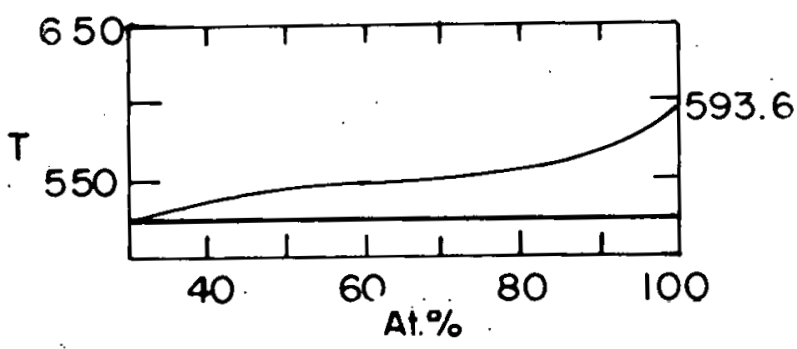

TEST DIAGRAM\#3

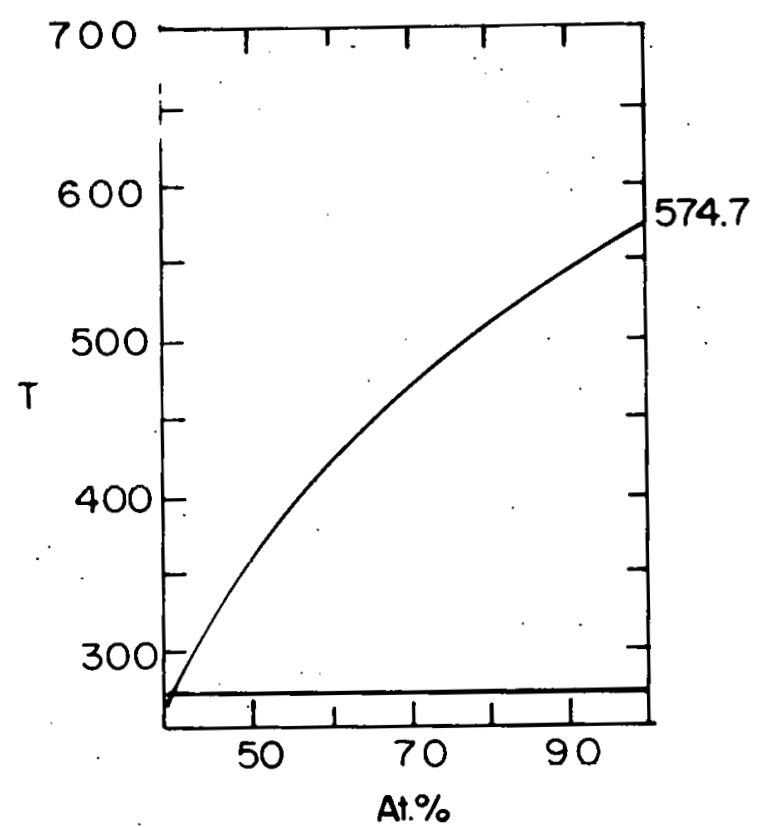

TEST DIAGRAM \#2

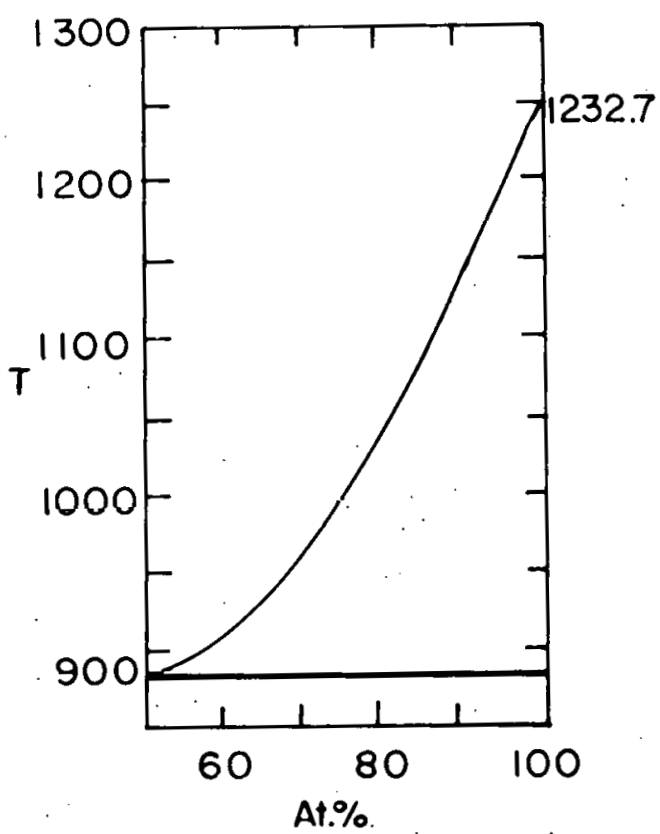

TEST DIAGRAM $\# 4$

Figure 2. Test diagrams plotted from third, fourth, and fifth degree power series polynomials 
Table 2. Coefficients of the power series polynomials used to create the test diagrams in Figure 2. The general form of the polynomial is $T=C_{1}+C_{2} x+C_{3} x^{2}+C_{4} x^{3}+C_{5} x^{4}+C_{6} x^{5}$

\begin{tabular}{lrrrrrr}
\hline \multirow{2}{*}{$\begin{array}{c}\text { Test } \\
\text { diagram }\end{array}$} & \multicolumn{6}{c}{ Coefficients } \\
\cline { 2 - 7 } & \multicolumn{1}{c}{$\mathrm{C}_{1}$} & \multicolumn{1}{c}{$\mathrm{C}_{2}$} & \multicolumn{1}{c}{$\mathrm{C}_{3}$} & \multicolumn{1}{c}{$\mathrm{C}_{4}$} & \multicolumn{1}{c}{$\mathrm{C}_{5}$} & $\mathrm{C}_{6}$ \\
\hline$\# 1$ & 315.3 & 4009.0 & -20504.9 & 37015.2 & -27107.0 & 7004.3 \\
$\# 2$ & -821.0 & 5260.3 & -8840.8 & 7234.8 & -2258.6 & 0 \\
$\# 3$ & 510.5 & -124.1 & 988.8 & -1658.3 & 876.7 & 0 \\
$\# 4$ & 1413.1 & -2402.9 & 3159.2 & -936.7 & 0 & 0 \\
\hline
\end{tabular}

ine the various numerical methods. The approximate precision of the test diagram data was \pm 1.5 degrees. The first and second derivatives of the four test curves were determined by each of the three previously discussed methods. The results are presented in Table 3.

Comparison of the various methods given in Table 3 indicated that the graphical derivative method was the least sensitive to the errors in the original data. This was undoubtedly due to the continual smoothing of the data in this method. The gross errors in the second derivatives obtained by the method of undetermined coefficients were not surprising since only five data points are utilized by Equations III-12 and III-13. The interpolation polynomial, although not as effective as the graphical method, gave fairly good results. The undetermined coefficient and interpolation polynomial 
Table 3. Comparison of methods for obtaining derivatives

\begin{tabular}{|c|c|c|c|c|c|c|c|}
\hline \multirow[t]{2}{*}{$\begin{array}{l}\text { Test } \\
\text { curve }\end{array}$} & \multicolumn{2}{|c|}{$\begin{array}{l}\text { Undetermined } \\
\text { coefficients }\end{array}$} & \multicolumn{2}{|c|}{$\begin{array}{c}\text { Interpolating } \\
\text { polynomials }\end{array}$} & \multicolumn{2}{|c|}{$\begin{array}{l}\text { Graphical } \\
\text { method }\end{array}$} & $\begin{array}{c}\text { Actual } \\
\text { derivative }\end{array}$ \\
\hline & $\frac{d T}{d x}$ & $\%$ error & $\frac{\mathrm{dT}}{\mathrm{dx}}^{\text {First }}$ & $\begin{array}{l}\text { derivative } \\
\% \text { error }\end{array}$ & $\frac{\mathrm{dT}}{\mathrm{dx}}$ & $\%$ error & $\frac{\mathrm{dT}}{\mathrm{dx}}$ \\
\hline$\# 1$ & +558 & -12.5 & +573 & -10.2 & +582 & -8.8 & +638.4 \\
\hline$\# 2$ & +210 & -15.6 & +212 & -14.8 & +232 & -6.7 & +248.8 \\
\hline$\# 3$ & +475 & 23.2 & +362 & -6.0 & +412 & 7.0 & +385.5 \\
\hline \multirow[t]{2}{*}{$\# 4$} & +1089 & 1.5 & +1090 & -1.3 & +1094 & -1.0 & +1105.4 \\
\hline & $\begin{array}{l}\text { Ave. } \\
\text { error }\end{array}$ & $13.2 \%$ & $\begin{array}{l}\text { Ave. } \\
\text { error }\end{array}$ & $8.1 \%$ & $\begin{array}{l}\text { Ave. } \\
\text { error }\end{array}$ & $5.9 \%$ & \\
\hline
\end{tabular}

\begin{tabular}{|c|c|c|c|c|c|c|c|}
\hline \multicolumn{8}{|c|}{ Second derivative } \\
\hline & $\frac{d^{2} T}{d x^{2}}$ & $\%$ error & $\frac{d^{2} T}{d x^{2}}$ & $\%$ error & $\frac{\mathrm{d}^{2} \mathrm{~T}}{\mathrm{dx} \mathrm{x}^{2}}$ & $\%$ error & $\frac{d^{2} T}{d x^{2}}$ \\
\hline$\# 1$ & -6700 & -38.5 & -5600 & 36.0 & -4500 & 9.3 & -4116 \\
\hline$\# 2$ & -1950 & +41.7 & -1840 & 33.7 & -1475 & 7.2 & -1376 \\
\hline$\# 3$ & +6767 & 165 & +2084 & -34.2 & +3160 & 23.0 & +2549 \\
\hline \multirow[t]{2}{*}{$\# 4$} & +450 & -35.5 & +560 & $-20 \cdot 0$ & +720 & 3.1 & +698.2 \\
\hline & $\begin{array}{l}\text { Ave. } \\
\text { error }\end{array}$ & $70.2 \%$ & $\begin{array}{l}\text { Ave. } \\
\text { error }\end{array}$ & $31.0 \%$ & $\begin{array}{l}\text { Ave. } \\
\text { error }\end{array}$ & $10.6 \%$ & \\
\hline
\end{tabular}

${ }^{a}$ All derivatives are for the limiting value of $x=1.0$. 
methods could probably have benefited from the smoothing procedure used in the graphical method. However, the primary purpose for examining Equations III-12 through III-15 was to determine if the rather subjective procedure of graphical smoothing could be avoided. Since the graphical method resulted in the least amount of error in the derivatives, it was chosen as the alternate method of calculating the derivatives from the phase boundary data.

The major problem encountered with the utilization of the graphical derivative method during the preliminary testing was uncertainties in extrapolation and smoothing caused by random errors in the original data, When a rapid change in the curvature occurred in the region before $x_{A}^{\prime}=1.0$, it was sometimes difficult to ascertain the proper path of extrapolation. In test diagram \#3, for example, the curve rapidly bends upward near the endpoint. Figure 3 gives the $d T / d x$ versus $x$ and the $\mathrm{d}^{2} \mathrm{~T} / \mathrm{dx}^{2}$ versus $\mathrm{x}$ plots used to obtain the first and second derivatives of test diagram \#3. The error bars denote the uncertainties that would be experienced if the original $T$ versus $x$ data points were known to within \pm 1.5 degress. As can be seen from both Figure 3 and Table 3 , the second derivative was considerably overestimated because of the error in the last data point. In cases such as test diagram \#3, the use of a larger number of data points, especially in the region near the endpoint, would undoubtedly help to alleviate some of 


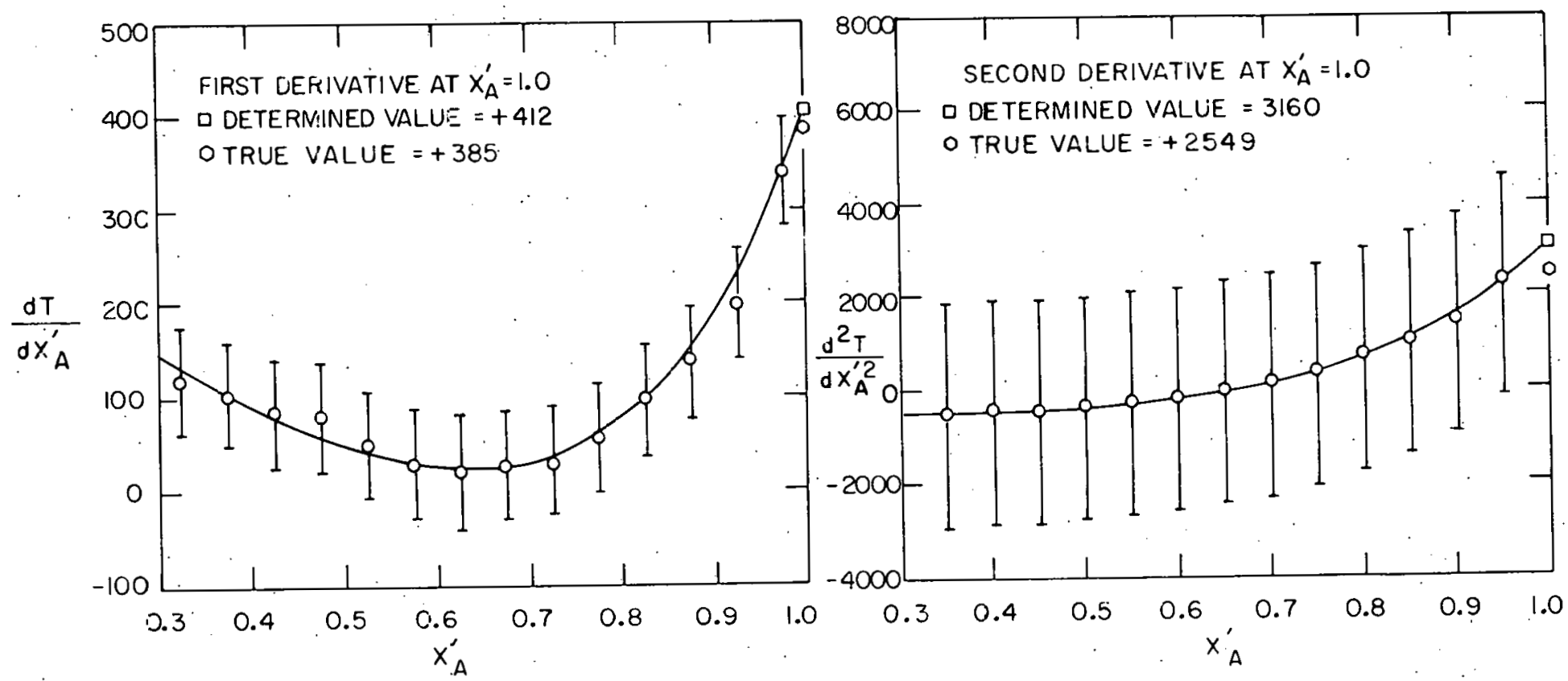

Figure 3. Flots used to determine the first and second derivatives of test diagram \#3. The error bars denote the propagation of error for $T$ versus $x_{A}^{i}$ data of $\pm 1.5^{\circ} \mathrm{C}$ accuracy. 
these uncertainties.

The size of the error bars in Figure 3 emphasize the need for smoothing in the graphical derivative method. Although formulas analogous to Equation III-16. which utilize more data points could be employed for the graphical method, the errors introduced by the use of this approximate equation are minor in comparison to the effects of the random errors in the original data. A study of the propagation of errors through the use of Equation III-16 revealed that an uncertainty of \pm 1.5 degrees in the $T$ versus $x$ plot would produce an uncertainty of \pm 2400 in the second derivative or an uncertainty of approximately \pm 2.0 in the self-interaction parameter. The actual error in the derivatives may be somewhat less than this, however, because of the continual smoothing employed in the method. 
IV. RESULTS AND DISCUSSION

In order to examine the usefulness of Equations I-I0 and II-39, $\varepsilon_{i}^{i}$ values for 22 non-ferrous liquid solutions were calculated from the liquidus and solidus phase boundaries of 13 binary phase diagrams and compared with the corresponding experimental $\varepsilon_{i}^{i}$ values of Dealy and Pehlke (11). Utilizing the graphical methods previously described section III A (see pages 32-33), Dealy and Pehlke have determined the binary and ternary interaction parameters of a number of non-ferrous liquid solutions from various sources of experimental activity coefficient data. Of the solutions examined by these authors, 22 possessed liquidus and solidus phase boundaries which were applicable to either Equation I-10 or II-39 and these 22 were selected for examination.

Equation I-10 was utilized in the calculations for phase boundaries possessing limited solid solubility while Equation II-39 was used for those possessing negligible solid solubility. The differential quantities required for the equations were determined by both the power series curve fitting method and the graphical derivative method (previously discussed in pages 40-55). The liquidus and solidus data were obtained from phase diagrams given in Constitution of Binary Alloys by Hansen and Anderko (20). The first and second supplements to Hansen's work by Elliott (13) and by Shunk (55) were also used when more recent or complete phase boundary data were avail- 
able. Thermodynamic information for the pure solvent components were obtained from Hultgren et al. (27).

The calculated values for the self-interaction parameters along with the observed values of Dealy and Pehlke (1i) are given in Table 4 . The $\varepsilon_{i}^{i}$ values obtained by the graphical derivative method are given in column 3 and the values obtained by the power series curve fitting method are given in column 4. The superscripts (c) and (d) in column 1 denote the use of Equations I-10 and II-39, respectively. Dealy and Pehlke obtained the particular values given in column 6 by graphically determining the slopes of experimental $1 \mathrm{n} \gamma_{\mathrm{i}}$ versus $x_{i}$ curves at $x_{i}=0$. The temperatures for the calculated values and those for Dealy and Pehlke's values are given in columns 5 and 7 , respectively.

In order to obtain an estimate of the uncertainties in the calculated phase diagram values, a statistical analysis was performed on the data using methods described by Bevington (4, pp. 92-122). The two sets of calculated values were each plotted versus the ohserved values of Dealy and Pehike and a linear least squares fit was performed on the curves. Assuming that the $\varepsilon_{i}^{i}$ values of Dealy and Pehlke are the "true" values of the self-interaction parameters, the standard deviation for each set of phase diagram values was then calculated using the following expression (4, p. 93):

$$
\sigma=\sqrt{\frac{1}{N-2} \sum_{k=1}^{N}\left(Y_{k}^{C}-Y_{k}^{L S}\right)^{2}}
$$


Table 4. Comparison of calculated self-interaction parameters with the observed parameters of Dealy and Pehlke

\begin{tabular}{|c|c|c|c|c|c|c|}
\hline \multirow[t]{2}{*}{ System } & \multirow{2}{*}{$\varepsilon_{i}^{i}$} & \multicolumn{2}{|c|}{$\varepsilon_{i}^{i}($ calculated $)$} & \multirow[t]{2}{*}{$\mathrm{T}{ }^{\circ} \mathrm{C}$} & \multicolumn{2}{|c|}{$\varepsilon_{i}^{i}($ observed $)$} \\
\hline & & $\begin{array}{l}\text { Graphicala } \\
\text { derivative }\end{array}$ & $\begin{array}{l}\text { Power series } \\
\text { curve fitting }\end{array}$ & & $\begin{array}{l}\text { Dealy and } \\
\text { Peh1ke (11) }\end{array}$ & $\mathrm{T}{ }^{\circ} \mathrm{C}$ \\
\hline$\frac{\mathrm{Ag}-\mathrm{Pb}}{\mathrm{Ag}-\mathrm{rich}} \mathrm{c}$ & $\varepsilon_{\mathrm{Pb}}^{\mathrm{Pb}}$ & +0.4 &.+2.1 & 960 & -0.4 & 1000 \\
\hline $\mathrm{Pb}-\mathrm{rich}{ }^{\mathrm{d}}$ & $\varepsilon_{\mathrm{Ag}}^{\mathrm{Ag}}$ & +1.1 & +2.8 & 327 & -0.6 & 1000 \\
\hline$\frac{A u-B i}{B i-r i c h} d$ & $\varepsilon_{\mathrm{Au}}^{\mathrm{Au}}$ & +5.6 & +4.5 & 271 & +2.1 & 700 \\
\hline $\mathrm{Au}-\mathrm{rich}$ & $\varepsilon_{\mathrm{Bi}}^{\mathrm{Bi}}$ & +2.4 & +4.0 & 1063 & +0.1 & 700 \\
\hline$\frac{A u-P b}{A u-r i c h} d$ & $\begin{array}{r}\mathrm{Pb} \\
\varepsilon_{\mathrm{Pb}}\end{array}$ & +0.5 & +4.1 & 1063 & +5 & 600 \\
\hline $\mathrm{Pb}-\mathrm{rich}$ & $\varepsilon_{\mathrm{Au}}^{\mathrm{Au}}$ & +1.1 & +1.1 & 327 & +3.2 & 600 \\
\hline
\end{tabular}

${ }^{a}$ Statistical comparison of the graphical derivative values with the values of Dealy and Pehlke yield $\sigma=3.69, \varepsilon_{i}^{1}($ graphical $)= \pm 6.1, r=0.575$, and a $1 \%$ probability of no correlation.

${ }^{b}$ Statistical comparison of the power series curve fitting values with the values of Dealy and Pehlke give $\sigma=2.37, \varepsilon+1$ (power series) $= \pm 3.9, r=0.644$, and a $0.5 \%$ probability of no correlation.

${ }^{c}$ Denotes the use of Equation I-10 to calculate $\varepsilon_{B}^{B}$ (limited solid solubility).

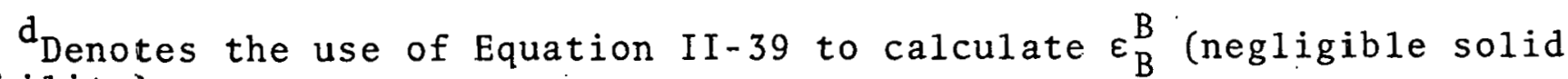
solubility). 
Table 4. (Continued)

\begin{tabular}{|c|c|c|c|c|c|c|}
\hline \multirow[t]{2}{*}{ System } & \multirow[t]{2}{*}{$\varepsilon_{i}^{i}$} & \multicolumn{2}{|c|}{$\varepsilon_{i}^{i}($ calculated $)$} & \multirow[t]{2}{*}{$\mathrm{T}{ }^{\circ} \mathrm{C}$} & \multicolumn{2}{|c|}{$\varepsilon_{i}^{i}($ observed) } \\
\hline & & $\begin{array}{l}\text { Graphicala } \\
\text { derivative }\end{array}$ & $\begin{array}{l}\text { Power series } \\
\text { curve fitting }\end{array}$ & & $\begin{array}{l}\text { Dealy and } \\
\text { Pehlke }(11)\end{array}$ & $\mathrm{T}{ }^{\circ} \mathrm{C}$ \\
\hline$\frac{\mathrm{Au}-\mathrm{T} 1}{\mathrm{Au}-\mathrm{rich}} \mathrm{c}$ & $\varepsilon_{\mathrm{T} 1}^{\mathrm{T} 1}$ & -1.5 & -2.2 & 1063. & +0.1 & 700 \\
\hline Tl-rich & $\varepsilon_{\mathrm{Au}}^{\mathrm{Au}}$ & $+1: 2$ & +1.3 & 302 & +2.6 & 700 \\
\hline$\frac{\mathrm{Bi}-\mathrm{Cd}}{\mathrm{Bi}-\mathrm{r} i \mathrm{ch}} \mathrm{d}^{\mathrm{d}}$ & $\varepsilon_{\mathrm{Cd}}^{\mathrm{Cd}}$ & +1.1 & +0.2 & 271 & -1.22 & 500 \\
\hline$C d-r i c h d$ & $\varepsilon_{\mathrm{Bi}}^{\mathrm{Bi}}$ & +0.7 & +1.0 & 321 & -6.5 & 500 \\
\hline$\frac{\mathrm{Bi}-\mathrm{Pb}}{\mathrm{Bi}-\mathrm{rich}} \mathrm{c}$ & $\varepsilon \frac{\mathrm{Pb}}{\mathrm{Pb}}$ & +7.0 & +1.7 & 271 & +1.95 & 271 \\
\hline$\frac{\mathrm{Bi}-\mathrm{Sn}}{\mathrm{Bi}-\mathrm{rich}} \mathrm{d}$ & $\varepsilon \stackrel{S n}{S n}$ & +0.9 & +0.3 & 271 & -0.0056 & 330 \\
\hline$\frac{B i-T 1}{B i-r i c h} d$ & $\Xi_{\mathrm{T} 1}^{\mathrm{T} 1}$ & $+\mathfrak{3} .2$ & +5.1 & 271 & +3.22 & 270 \\
\hline$\frac{\mathrm{Cd}-\mathrm{Pb}}{\mathrm{Cd}-\mathrm{rich}}$ & $\begin{array}{r}\mathrm{Pb} \\
\varepsilon_{\mathrm{Pb}}\end{array}$ & -5.4 & -4.8 & 321 & -4.56 & 500 \\
\hline $\mathrm{Pb}-\mathrm{rich}{ }^{\mathrm{C}}$ & $\varepsilon_{\mathrm{Cd}}^{\mathrm{Cd}}$ & -3.9 & -2.9 & 327 & -2.6 & 500 \\
\hline
\end{tabular}


Table 4. (Continued)

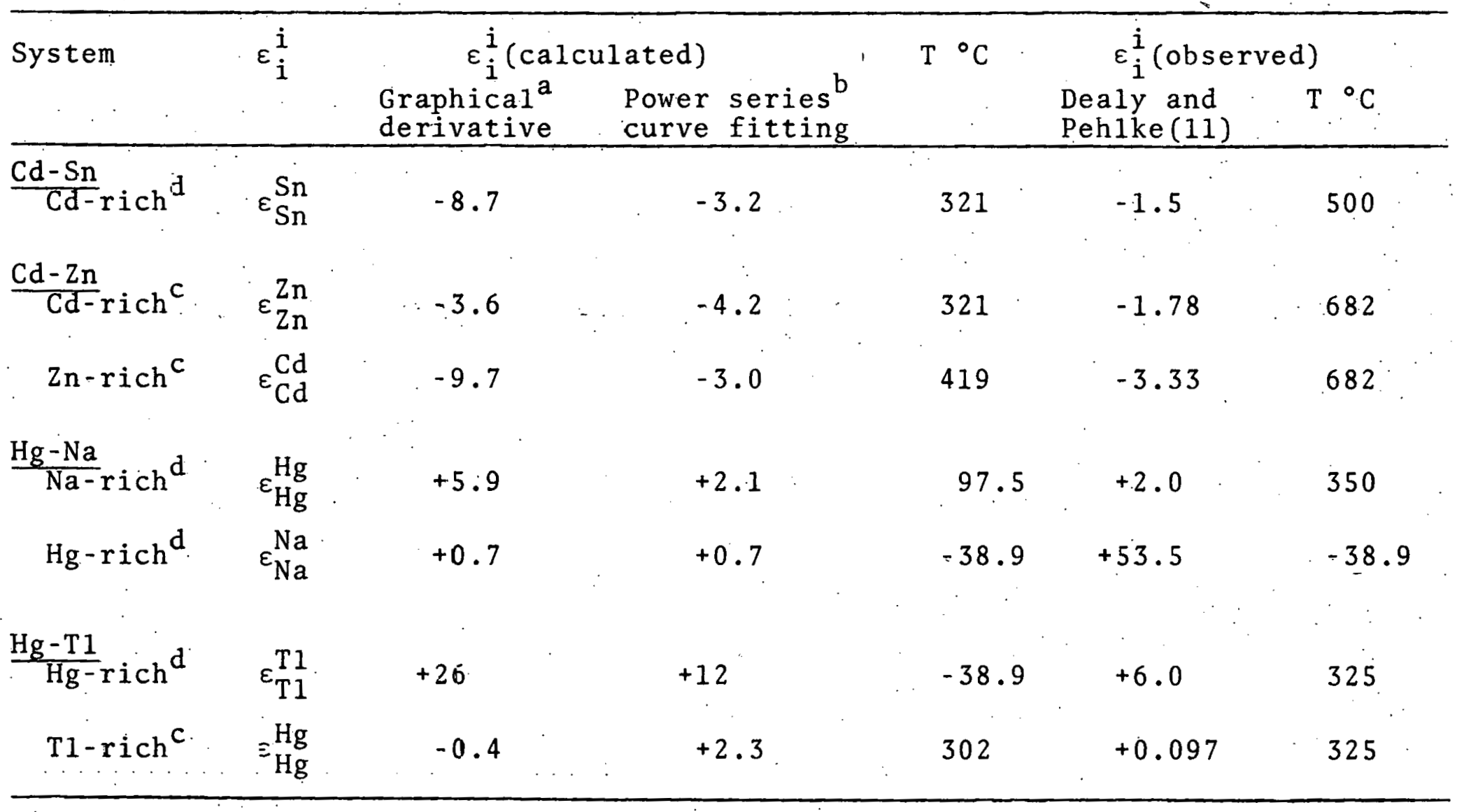


$\mathrm{N}$ is the number of observations, $\mathrm{Y}_{\mathrm{k}}^{\mathrm{C}}$ is the particular calculated phase diagram value of $\varepsilon_{i}^{i}$ and $\mathrm{Y}_{\mathrm{K}}^{\mathrm{LS}}$ is the value obtained from the least squares line. $Y_{k}^{L S}=a+b x_{k}$, where $x_{k}$ is the $\varepsilon_{i}^{i}$ value given by Dealy and Pehlke (11). Assuming a Gaussian distribution in the random errors of the calculated $\varepsilon_{i}^{i}$ values and assuming a confidence level of $90 \%$, the error limits for the calculated values are given by

$$
-1.64 \sigma \leq \varepsilon_{i}^{i}(\text { calculated }) \leq+1.64 \sigma
$$

A value for the linear correlation coefficient was calculated for both sets of phase diagram values. To obtain the coefficient, a linear least squares fit of $x_{k}$ versus $Y_{k}^{C}$ was performed on the data to obtain a value for $b^{\prime}$, the slope of the reciprocal line $x_{k}=a^{\prime}+b^{\prime} Y_{k}^{c}$. Here $x_{k}$ is considered a function of $Y_{k}^{c}$ whereas in the foregoing relation $Y_{k}^{C}$ is considered a function of $x_{k}$. The linear correlation coefficient is defined

$$
r=\sqrt{b b^{1}}
$$

A value of $r=0$ indicates no correlation and a value of $r=1$ indicates complete correlation (4, p. 121). While it would be difficult to obtain the probability of correlation without further statistical information about the individual data points, a percent probability of no correlation betwcen the calculated and observed $\varepsilon_{i}^{i}$ values is easily obtained from graphical information given by Bevington (4, p. 312). If the probability of no correlation is small, then there is a good 
probability that the data are correlated (4, p. 122).

The values of $\sigma$ obtained for the various calculation methods along with values for the percent probability of no correlation and the linear correlation coefficients are given in footnotes to Tables 4 and 6 .

Twenty of the 22. sets of $\varepsilon_{i}^{i}$ values given in Table 4 were used for the statistical analysis. The values for $\varepsilon_{\mathrm{Na}}^{\mathrm{Na}}$ of the $\mathrm{Hg}-\mathrm{Na}$ system and $\varepsilon_{\mathrm{T} 1}^{\mathrm{T} 1}$ of the $\mathrm{Hg} \times \mathrm{T} 1$ system were excluded because of the exceedingly large disagreement displayed between the values for the various procedures. The disagreements are probably the result of inadequate phase boundary data since, in both cases, the liquidus curves utilized for the calculations were less than 10 atomic percent in length.

The statistical comparison of the graphical derivative values with the values of Dealy and Pehlke gives a value of $\sigma=3.69$ for the standard deviation with a resultant confidence interval of $\varepsilon_{i}^{i}$ (graphical) $= \pm 6.1$ at a $90 \%$ confidence level. The linear correlation coefficient is $r=0.575$, indicaling that there is only a $1 \%$ probability that the graphical values are uncorrelated with the values of Dealy and Pehlke. Direct examination of the values in Table 4 re: veals that 13 of the 22 graphical derivative values are in agreement to within \pm 2.5 of the values of Dealy and Pehilke with 6 of the 22 values in disagreement by more than \pm 4.0 . Comparison of power series curve fitting values with 
those of Dealy and Pehlke indicates a somewhat closer agreement than is experienced with the graphical derivative values. The power series curve fitting values yield $\sigma=2.37$ for the standard deviation with a confidence interval of $\varepsilon_{i}^{i}$ (power series) $= \pm 3.9$. The linear correlation coefficient is $r=$ 0.644 with only a $0.5 \%$ probability of no correlation. Direct examination of the values given in Table 4 indicates that of the 22 pairs of values, 17 agree to within \pm 2.5 with 4 of. the values in disagreement by more than \pm 4.0 .

Although the observed values of Dealy and Pehlke have been assumed in the analysis to be the "true" values, they are somewhat less than ideal for comparison purposes. Except for two cases $\left(\varepsilon_{\mathrm{Pb}}^{\mathrm{Pb}}\right.$ of the $\mathrm{Bi}-\mathrm{Pb}$ system and $\varepsilon_{\mathrm{Na}}^{\mathrm{Na}}$ of the $\mathrm{Hg}-\mathrm{Na}$ system), all of the values of Dealy and Pehlke are for different temperatures than the phase diagram values. The calculated phase diagram values are necessarily for the temperature of fusion of the pure solvent component while the values of Dealy and Pehlke are for temperatures above the solvent melting temperature. For most solutions, the value of $\varepsilon_{i}^{i}$ does not change a great deal over a short temperature span. However, over a temperature span of several hundred degrees, the value of $\varepsilon_{i}^{i}$ may, in some cases, change as much as \pm 2.0 depending on the system in question (11). The temperature differences for the calculated and observed values are over $400^{\circ} \mathrm{C}$ for several of the systems given in Table 4. Furthermore some of their values were obtained by extrapolating (thermodynamic data for 
fairly concentrated solutions) over a composition range as large as 10 atomic percent to obtain the value for infinite dilution. Consequently the error limits obtained in the statistical analysis are probably somewhat larger than would be the case if "true" or errorless values were available for comparison.

The empirical $\Delta \bar{G}_{i}^{X S}=f\left(T, x_{A}\right)$ representation defined by Equation III-4,

$$
\Delta \overline{\mathrm{G}}_{\mathrm{A}}^{\mathrm{XS}}=\left(1-\mathrm{x}_{\mathrm{A}}\right)^{2}\left[(\alpha+\beta T)+\left(\alpha^{\prime}+\beta^{\prime} \mathrm{T}\right) \mathrm{x}_{\mathrm{A}}+\left(\alpha^{\prime \prime}+\beta^{\prime \prime} \mathrm{T}\right) \mathrm{x}_{\mathrm{A}}^{2}\right]
$$

was also used to obtain $\varepsilon_{i}^{i}$ values for comparison with the calculated phase diagram values. The self-interaction parameters are calculated from the differentiated forms of this expression given by Equations III-5 and III-6. Chiotti, Simmons and Kateley ( 8 ) have determined the coefficients of Equation III-4 for a number of binary systems from experimental activity data given by Hultgren, Orr, Anderson and Kelley (28). The coefficients for Equation III-4 are given in Table 5 along with values for the self-interaction parameter calculated by means of Equations III-5 and III-6.

Eight of the solutions given in Table 5 correspond to calculated phase diagram values given in Table 4 and these 8 are compared with the phase diagram values in Table 6 . Since Equation III-4 is a function of temperature as well as composition, it is possible to calculate $\varepsilon_{i}^{i}$ values from the coefficients given in Table 5 at the same temperatures as the phase 
Table 5. Coefficients for the empirical equation

$$
\Delta \overline{\mathrm{G}}_{\mathrm{A}}^{\mathrm{XS}}=\left(1-\mathrm{X}_{\mathrm{A}}\right)^{2}\left[(\alpha+\beta \mathrm{T})+\left(\alpha^{\prime}+\beta^{\prime} \mathrm{T}\right) \mathrm{X}_{\mathrm{A}}+\left(\alpha^{\prime \prime}+\beta^{\prime \prime} \mathrm{T}\right) \mathrm{X}_{\mathrm{A}}^{2}\right]
$$

and values for the self-interaction parameters calculated from the differentiated forms of the expressiona

\begin{tabular}{|c|c|c|c|c|c|c|c|c|c|}
\hline $\begin{array}{c}\text { Sys tem } \\
\text { A-B. }\end{array}$ & $\alpha$ & $\beta$ & $\alpha^{\prime}$ & $\beta^{\prime}$ & $\alpha^{\prime \prime}$ & $\beta^{\prime \prime}$ & $\varepsilon_{A}^{A}$ & $\varepsilon_{\mathrm{B}}^{\mathrm{B}}$ & $\mathrm{T}^{\circ} \mathrm{K}^{\mathrm{b}}$ \\
\hline $\mathrm{Ag}-\mathrm{Au}(\mathrm{s})$ & -4050 & 1.378 & -1614 & -0.043 & 23.4 & 0.064 & +2.67 & +5.69 & 800 \\
\hline $\mathrm{Ag}-\mathrm{Au}(\ell)$ & -3859 & $1.37 \varepsilon$ & -1576 & -0.043 & -35.3 & 0.064 & +0.88 & +2.67 & 1350 \\
\hline $\mathrm{Au}-\mathrm{Cu}(\mathrm{s})$ & -4813 & 0.002 & -6334 & 1.180 & 16368 & -1.928 & +2.89 & -6.55 & 720 \\
\hline $\mathrm{Au}-\mathrm{Ni}(\mathrm{s})$ & 6936 & -0.664 & 2953 & -14.780 & -8222 & 18.381 & -11.57 & -4.42 & 1150 \\
\hline $\mathrm{Au}-\mathrm{Sn}(\mathrm{s})$ & -8199 & -0.694 & -1479 & -8.419 & -18753 & 8.147 & +5.06 & +33.74 & 873 \\
\hline$A 1-S n(l)$ & 3499 & -1.90 & 10030 & -9.710 & -36.9 & 5.46 & -1.44 & -7.39 & 1000 \\
\hline $\mathrm{Bi}-\mathrm{Pb}(\ell)$ & -833 & -0.279 & -1593 & 0.543 & 2440 & -0.968 & +0.61 & +0.71 & 700 \\
\hline $\mathrm{Bi}-\mathrm{Sn}(\ell)$ & 92 & 0.427 & -63 & -0.498 & 142 & -0.141 & -0.88 & -1.07 & 608 \\
\hline $\mathrm{Bi}-\mathrm{T} 1(\ell)$ & -5489 & -0.211 & .5744 & -0.193 & -439 & -3.454 & +11.61 & +4.14 & 732 \\
\hline $\mathrm{Bi}-\mathrm{Zn}(\mathrm{l})$ & 7944 & -2.106 & -20650 & 0.232 & 18957 & -0.479 & -19.88 & -4.42 & 873 \\
\hline $\mathrm{Cd}-\mathrm{Ga}(\ell)$ & 3074 & -0.082 & -5881 & 3.201 & 10163 & -6.731 & -6.95 & -6.94 & 700 \\
\hline $\operatorname{Cd}-\operatorname{In}(\ell)$ & $1154^{\circ}$ & -0.273 & .365 & -1.686 & 1418 & 1.719 & -1.93 & -3.78 & 723 \\
\hline $\mathrm{Cd}-\mathrm{Pb}(\ell)$ & 2351 & -0.615 & 163 & -0.056 & 4218 & -2.696 & -2.36 & -5.37 & 773 \\
\hline
\end{tabular}

${ }^{a}$ Values for the coefficients were taken from a paper by Chiotti, Simmons and Kateley (.8).

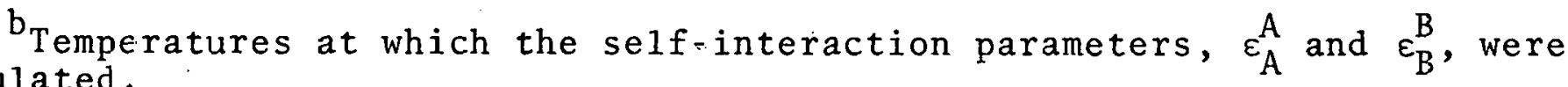
calculated. 
Table 5. (Continued)

\begin{tabular}{|c|c|c|c|c|c|c|c|c|c|}
\hline $\begin{array}{l}\text { System } \\
\text { A-B }\end{array}$ & $\alpha$ & $\beta$ & $\dot{\alpha}^{\prime}$ & $\beta^{\prime}$ & $\alpha^{\prime \prime}$ & $\beta^{\prime \prime}$ & $\varepsilon_{A}^{A}$ & $\varepsilon_{B}^{B}$ & $T^{\circ} K^{b}$ \\
\hline $\mathrm{Cd}-\mathrm{Sn}(\ell)$ & 1550 & -0.943 & -45 & 0.368 & 2380 & -2.687 & -0.96 & -1.67 & 773 \\
\hline $\mathrm{Cd}-\mathrm{T} 1(\ell)$ & 1817 & -0.529 & 460 & -1.116 & 3332 & -1.512 & -2.40 & -5.21 & 673 \\
\hline $\mathrm{Cd}-\mathrm{Zn}(\ell)$ & 2212 & $\div 0.270$ & $=2409$ & 3.393 & 3545 & -4.337 & -2.32 & -2.99 & 800 \\
\hline $\mathrm{Cu}-\mathrm{Ni}(\mathrm{s})$ & 2816 & 0.484 & -7186 & 3.752 & 4745 & -2.155 & -6.66 & -2.48 & 973. \\
\hline $\mathrm{Ga}-\mathrm{Zn}(\ell)$ & .. 2187 & -1.455 & -3809 & $2: 798$ & 2825 & -2.086 & -2.82 & -0.93 & 723 \\
\hline $\mathrm{Cu}-\mathrm{Pd}(\mathrm{s})$ & -9475 & 5.712 & -1764 & -9.392 & -5787 & 7.401 & -0.05 & +13.39 & 1000 \\
\hline $\mathrm{Hg}-\operatorname{In}(\ell)$ & -1744 & -0.735 & -2499 & 6.750 & 2574 & -6.936 & +5.29 & +4.81 & 433 \\
\hline $\mathrm{Hg}-\mathrm{K}(\ell)$ & -13404 & 15.116 & -31536 & 13.107 & 9957 & -35.069 & -12.58 & +65.57 & 600 \\
\hline $\operatorname{In}-\operatorname{Sn}(\ell)$ & -172 & 0.236 & 646 & -6.921 & -2062 & 5.934 & -3.08 & +2.82 & 773 \\
\hline $\operatorname{In}-\operatorname{Zn}(\ell)$ & 4173 & -1.235 & -5834 & -0.010 & $4289^{\circ}$ & 0.682 & -8.96 & -6.28 & 700 \\
\hline $\operatorname{In}-\mathrm{Pb}(\ell)$ & 834 & -.0 .647 & 386 & 0.501 & -212 & 0.283 & -0.06 & -1.65 & 673 \\
\hline$M g-C d(s)$ & -2375 & -1.921 & -19249 & 19.226 & 24146 & -21.507 & -1.83 & -0.95 & 543 \\
\hline $\mathrm{Mg}-\mathrm{Pb}(\ell)$ & .3299 & 0.342 & -20417 & 7.707 & 21 & 0.039 & -4.81 & +20.49 & 833 \\
\hline $\mathrm{Pb}-\mathrm{Sn}(\ell)$ & 1370 & 0.0 & -1037 & 0.0 & 1958 & 0.0 & -2.46 & -2.98 & 773 \\
\hline$F b-Z n(l)$ & 11858 & -5.539 & -24130 & 14.741 & 18403 & -11.752 & -13.01 & -4.10 & 926 \\
\hline$S b-S n(\ell)$ & -759 & -1.049 & -6124 & 8.680 & 4806 & -8.197 & +0.30 & +8.01 & 905 \\
\hline $\operatorname{Sn}-\operatorname{Zn}(l)$ & 52.98 & -3.585 & -120.60 & 7. .469 & 9.250 & -5.499 & -8.92 & -1.95 & 700 \\
\hline
\end{tabular}


Table 6. Comparison of calculated values for the selfinteraction parameters with values obtained from coefficients of Chiotti, Simmons, and Kateley (8)

\begin{tabular}{lcccc} 
System & $\varepsilon_{\mathrm{B}}^{\mathrm{B}}$ & $\varepsilon_{\mathrm{B}}^{\mathrm{B}}$ (calculated) & $\varepsilon_{\mathrm{B}}^{\mathrm{B}}$ (observed) \\
& $\begin{array}{c}\text { Graphical } \\
\text { method }\end{array}$ & $\begin{array}{c}\text { Power series } \\
\text { method }\end{array}$ & $\begin{array}{l}\text { Chiotti, } \\
\text { Simmons, \& } \\
\text { Kateley }\end{array}$ \\
\hline
\end{tabular}

$\underline{\mathrm{Bi}-\mathrm{Pb}}$

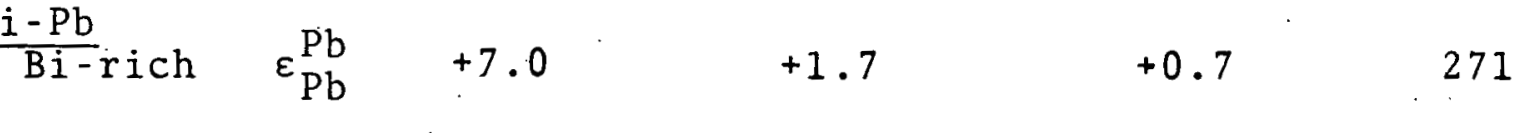

$\underline{B i-S n}$

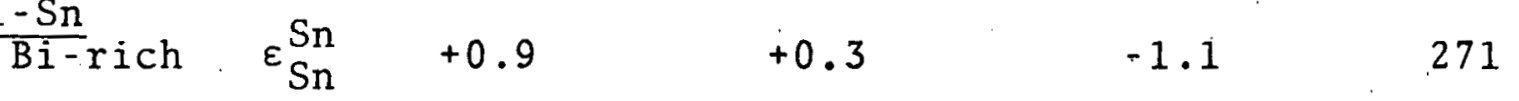

$\underline{\mathrm{Bi}-\mathrm{T} 1}$

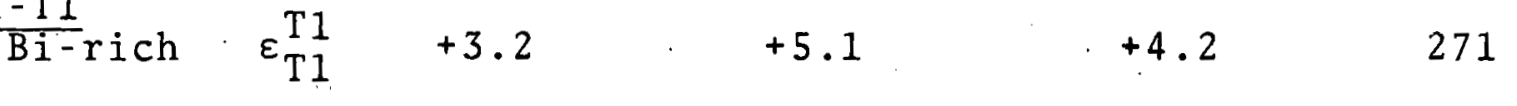

$\underline{\mathrm{Cd}-\mathrm{Pb}}$

$\begin{array}{lllll}\mathrm{Cd}-\mathrm{Pb} & & -4.8 & -8.0 \\ \mathrm{~Pb}-\mathrm{rich} & \varepsilon_{\mathrm{Pb}}^{\mathrm{Pb}} & -5.4 & -2.9 & -3.2\end{array}$

321

327

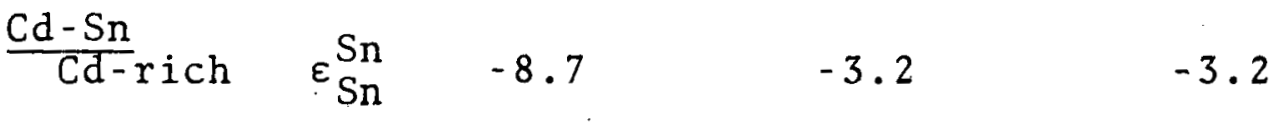

321

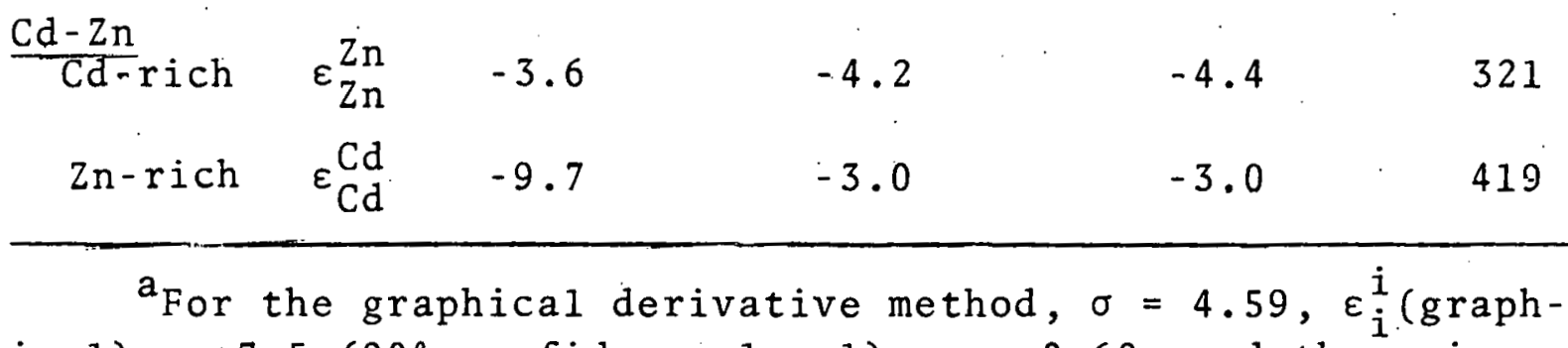
ica 1$)= \pm 7.5(90 \%$ confidence leve1), $r=0.68$, and there is a $5 \%$ probability of no correlation.

${ }^{b}$ For the power series method, $\sigma=1.09, \varepsilon_{i}^{i}$ (power series) $=$ $1.8, r=0.95$, and there is a $0.1 \%$ probability of no correlation. 
diagram values. The data in Table 6 were submitted to the same statistical analysis previously utilized with the values of Dealy and Pehlke.

Comparison of the graphical derivative values with those obtained from the coefficients of Chiotti, Simmons, and Kateley gives a value of $\sigma=4.59$ for the standard deviation and a resultant $90 \%$ confidence interval of $\varepsilon_{i}^{i}$ (graphical) $=$ +7.52. The linear correlation coefficient is $r=0.68$ and there is a $5 \%$ probability of no correlation. A direct examination of the graphical derivative values given in column 3 of Table 6 reveals that only 4 of $8 \varepsilon_{i}^{i}$ values agree to within \pm 2.5 with the values of Chiotti, Simmons and Kateley. Three of the values disagree by more than \pm 4.0 .

The power series values are in considerably better agreement with a standard deviation of $\sigma=1.09$ and a $90 \%$ confidence interval of $\varepsilon_{i}^{i}$ (power series) $= \pm 1.8$. The linear correlation coefficient is $r=0.95$ with a resultant probability of no correlation of only $0.1 \%$. Direct comparison of the values in columns 4 and 5 of Table 6 shows that 7 of the 8 values agree to within \pm 1.5 and 4 of the 8 agree to within \pm 0.5 . The only power series value in considerable variance is $\varepsilon_{\mathrm{Pb}}^{\mathrm{pb}}$ of the $\mathrm{Cd}-\mathrm{Pb}$ system.

In order to verify the calculations based on the diagrams of Hansen and Anderko (20), three of the solutions in Table 4 were redetermined utilizing precise phase boundary data ob- 
tained from the literature. $\varepsilon_{S n}^{S n}$ of the $C d-S n$ system and $\varepsilon_{C d}^{C d}$ and $\varepsilon_{\mathrm{Pb}}^{\mathrm{Pb}}$ of the $\mathrm{Cd}-\mathrm{Pb}$ system were calculated. Data points for both the $\mathrm{Cd}-$ rich and the $\mathrm{Pb}-\mathrm{rich}$ liquidus curves of the $\mathrm{Cd}-\mathrm{Pb}$ system were obtained from the work of Schürmann (54), Jänecke (29), and Barlow (3). The Pb-rich liquidus was based solely on Schürmann's work. The Pb-rich solidus was obtained from Rollason and Hysel (49). Schürmann's data have an estimated precision of $\pm 0.3^{\circ} \mathrm{C}$ and his data were weighted the heaviest of the three authors in the examination of the cd-rich liquidus. Data points for the Cd-rich liquidus of the Cd-Sn system were obtained from the work of Hanson and Pe11-Walpole (21) and also from Schleicher (53). Hanson and Pel1-Walpoles' data have an estimated precision of $\pm 1.0^{\circ} \mathrm{C}$ and the estimated precision of Schleicher's data is about $\pm 1.5^{\circ} \mathrm{C}$.

The experimental data points were used to calculate the self-interaction parameters by both the graphical derivative method and the power series curve fitting method. In preparation for the graphical derivative method, the data points were plotted on an enlarged scale and a smooth curve was carefully drawn through the points. The scale was large enough to per: mit the smoothed $T$ versus $x_{A}^{\prime}$ curves to be read with a precision of $\pm 0.2^{\circ} \mathrm{C}$. Points were read from the smoothed curves at $x_{A}^{\prime}=0.02$ increments and submitted to the graphical derivative method. The actual experimental data points were used for the power series curve fitting method. The data points 
were weighted according to the degree of precision with the most precise points receiving the most weight. The results of the calculations are given in Table 7 along with the corresponding, values obtained by using the diagrams of Hansen and Anderko (20). The values of Dealy and Pehlke (11) and of Chiotti, Simmons and Kateley (8) are also given for reference. Comparison of the literature values with the values obtained by the various other procedures show that, at least in these 3 cases, the diagrams given in Constitution of Binary Alloys are fairly faithfully reproduced and that meaningful values for the self-interaction parameters can be obtained from these diagrams. A considerable improvement is seen in the agreement of $\varepsilon_{\mathrm{Sn}}^{\mathrm{Sn}}$ (graphical) of the Cd-Sn with the thermodynamic values. The urcertainties in smoothing and extrapolation previously encountered with the diagrams of Hansen and Anderko were considerably reduced by the use of the precise liquidus and solidus data. The improvement in this graphical value is indicative of the need for precise, reliable phase diagram data in the phase diagram calculations.

The linear correlation coefficients and the percent probabilities of no correlation given in Tables 4 and 6 strongly indicate that the phase diagram values are correlated with the two sets of $\varepsilon_{i}^{i}$ values obtained from thermodynamic measurements. While the exact degree of correlation cannot be determined without further study, the results of the analysis demonstrate that Equation I-10 and II-39 can be used to calcu- 
Table 7. Comparison of the self-interaction parameters calculated from various literature data with the calculated values from the diagrams of Hansen et a1. and the two sets of observed values

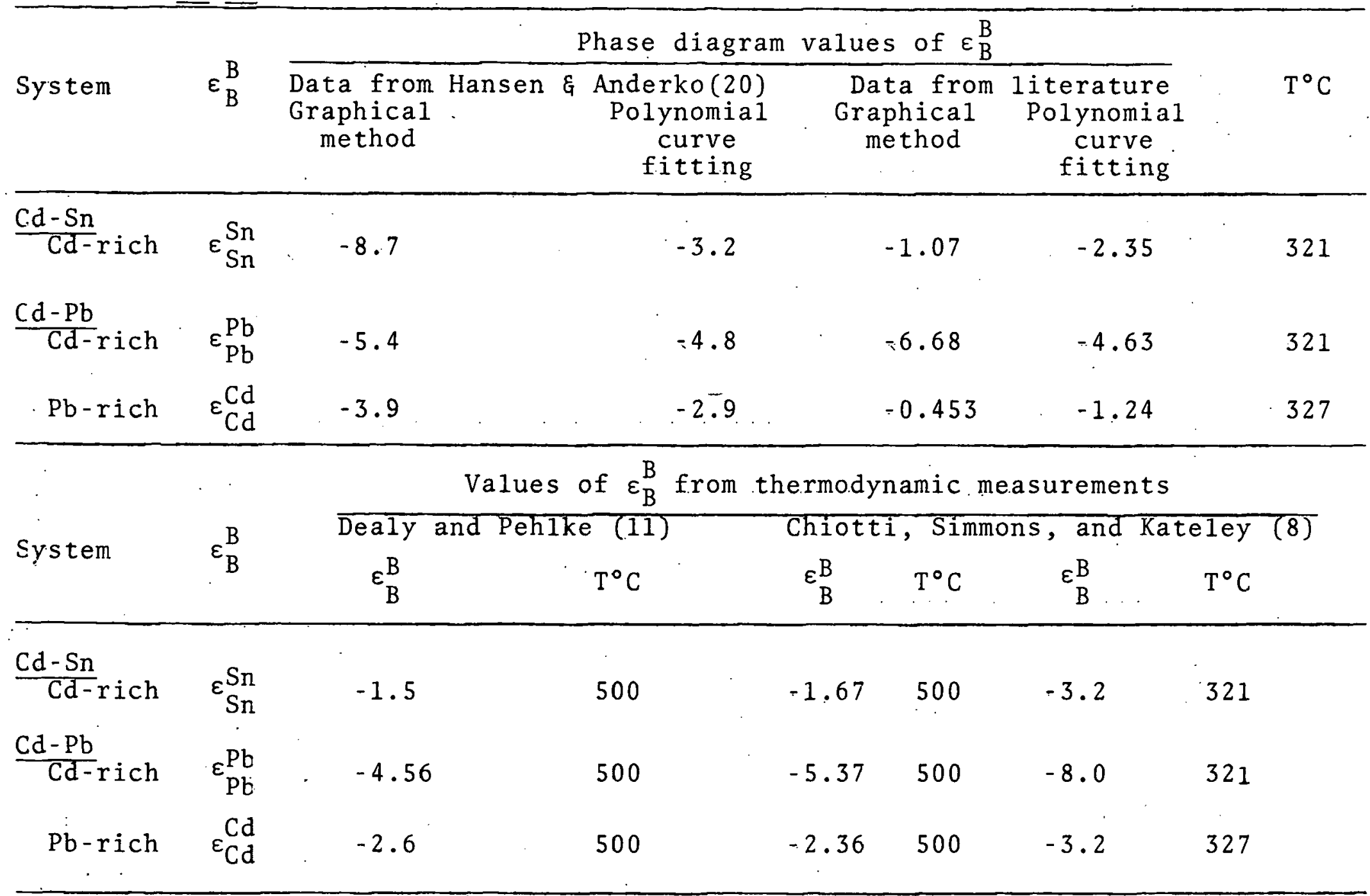


late the selfinteraction parameters from binary phase boundary data.

The results of the statistical analysis show that the power series curve fitting method is the better of the two methods for determining the derivatives of phase boundaries data. In both the comparison with the values of Dealy and Pehlke and the comparison with the values of Chiotti, Simmons and Kateley, the graphical derivative values possess a much larger standard deviation and confidence interval than the power series values. 


\section{SUMMARY}

The feasibility of utilizing Equations I-10 and II-39 to calculate self-interaction parameters from binary phase diagrams has been examined. Several numerical methods for determining first and second derivatives have been explored for their potential usefulness in calculating the differential quantities required by these equations. Two of these methods, the power series curve fitting method and the graphical derivative method, were used throughout the examination to calculate the first and second derivatives of the phase boundary data. The results of the examination show that the power series curve fitting method is the more suitable of the two methods for calculations involving data of limited precision.

The self-interaction parameters of 22 non-ferrous solutions were calculated from the binary phase diagrams of Hansen and Anderko and compared with various $\varepsilon_{i}^{i}$ values obtained from experimental activity coefficient measurements. In order to verify the calculations based on these diagrams, three of the 22 values were recalculated using various phase boundary data obtained from the literature. Statistical analysis of the data indicates that Equations I-10 and II-39 can be used to calculate values for the self-interaction parameter from binary phase boundary data. The confidence intervals for these calculations are $\varepsilon_{\dot{i}}^{i}$ (graphical) $= \pm 6.1$ for the graphical derivative method and $\varepsilon_{i}^{i}$ (power series) $= \pm 3.9$ for the power 
series curve fitting method. It is anticipated that the use of precise phase boundary data will lead to a decrease in the uncertainties of these values, 


\section{VI, LITERATURE CITED}

1. Bale, C. W. and Pelton, A. D. 1974. Mathematical representation of thermodynamic properties in binary systems and solution of Gibbs-Duhem equation. Metallurgical Transactions 5: 2323-2337.

2. Bale, C. W. and Pelton, A, D. 1975. Series representations of the the rmodynamic properties of solutions. Canadian Metallurgical Quarteriy 14, number 3: 213-219.

3. Barlow, W. E. 1910. The binary and ternary alloys of cadmium, bismuth, and lead. Journal of the American Chemical Society 32: 1390-1412.

4. Bevington, P. R. 1969. Data reduction and error analysis for the physical sciences. McGraw-Hill Book Company, New York, N.Y.

5. Boyle, M. L., Van Tyne, C. J. and Tarby, S. K. 1976. Computer analysis and synthesis of solution thermodynamics and phase diagrams. Pages 187-197. In R. J. Arsenault, J. R. Beeler, Jr. and J.A. Simmons, eds. Nuclear Metallurgy 20, part 1. Proceedings of the 1976 International Conference on Computer Simulation for Materials Applications; Aprì 19-21, 1976. National Bureau of Standards, Gaithersburg, Maryland.

6. Chiotti, P. 1974. New integration of the Gibbs-Duhem equation and application of phase relations and thermodynamics to fuel reprocessing. Journal of Nuclear Materials 51: 178-194.

7. Chiotti, P. C. Private communication. Ames Laboratory of U.S.E.R.D.A., Ames, Iowa. March, 1975.

8. Chiotti, P., Simmons, M. F. and Kateley, J. A. 1969. Calculation of thermodynamic properties from binary phase diagrams. Pages 659-688, in P. Chiotti, ed. Symposium on reprocessing of nuclear fuels, Nuclear Metallurgy, Vol. 15. USAEC Report CONF-690801 (National Technical Information Service, IJ.S. Department of Commerce, Springfield, Va. 22151).

9. Chipman, J. 1955. Atomic interaction in molten alloy steels. Journal of the Iron and Steel Institute 180, Part 2: 97-106. 
10. Darken, L. S. 1967. Thermodynamics of ternary metallic solutions. Transactions of the Metallurgical Society of AIME 239: 90:96.

11. Dealy, J. M. and Pehlke, R. D. 1963. Interaction parameters in dilute molten alloys: Transactions of the Metallurgical Society of AIME 227: 88-94.

12. Denbigh, J. 1971. The principles of chemical equilibrium. Third Edition. Cambridge University Press, Cambridge.

13. El1iott, R. P. 1965. Constitution of binary alloys. First supplement. McGraw-Hill Book Company, Inc., New York, N.Y.

14. Eyring, H. 1936. Viscosity, plasticity, and diffusion as examples of absolute reaction rates. Journal of Chemical Physics 4: 83-291.

15. Gerald, C. F. 1970. Applied numerical analysis. Addison-Wesley Publishing Company, Reading, Mass .

16. Glasstone, S. 19.49. Thermodynamics for chemists. Fourth Edition. D. Van Nostrand Company, Inc., New York, N.Y.

17. Gluck, J.V. and Pehlke, R. D. 1965. The effect of silver on the activity of zinc in dilute solution with molten bismuth. Transactions of the Metallurgical Society of AIME 233: 233-240.

18. Guggenheim, E. A. 1967. Thermodynamics. Fifth edition. North Holland Publishing Co., Amsterdam.

19. Hadrys, H. G., Frohberg, M. G. and E1110te, J, F. 1970 . Activities in the liquid $\mathrm{Fe}-\mathrm{Cr}-\mathrm{C}$ (sat), Fe-P-C(sat), and $\mathrm{Fe}-\mathrm{Cr}-\mathrm{P}$ systems at $1600^{\circ} \mathrm{C}$. Metallurgical Transactions 1: $1867-1874$.

20. Hansen, M. and Anderko, K. 1958. Constitution of binary alloys. Second edition. McGraw-Hill Book Company, Inc., New York, N.Y.

21. Hanson, D. and Pell-Walpole, W. T, 1935. The constitution and properties of cadmium-tin alloys. Journal of the Institute of Metals $56: 165=189$.

22. Hardy, H. K. 1953. A "sub-regular" solution model and its application to some binary alloy systems. Acta 
Metallurgica 1: 202-209.

23. Hatsopoulos, G. N. and Keenan, J. H. 1965. Principles of general thermodynamics, Pirst edition. John Wiley and Sons, Inc., New York, N.Y.

24. Hiskes, R. and Tiller, W. A. 1967-1968. Generation of chemical potentials by analysis of phase diagrams, Part I. Materials Science and Engineering 2: 320-330.

25. Hiskes, R. and Tiller, W. A. 1969. Generation of chemical potentials by analysis of phase diagrams, Part II: Isomorphous systems. Materials Science and Engineering 4: $163-172$.

26. Hiskes, R. and Tiller, W. A. 1969. Generation of chemical potentials by analysis of phase diagrams, Part III: Eutectic systems. Materials Science and Engineering 4: $173-184$.

27. Hultgren, R., Desai, P. D., Hawkins, D. T., Gleiser, M., Kelley, K. K. and Wagman, D. D. 1973. Selected values of the thermodynamic properties of the elements. American Society for Metals, Metals Park, Ohio.

28. Hultgren, R., Orr, R. L., Anderson, P. D. and Kelley, K. K. 1963. Selected values of thermodynamic properties of metals and alloys. John Wiley and Sons, Inc., New York, N.Y.

29. Jänecke, E. 1907. Das ternäre System Pb-Cd-Hg. Zeitschrift für Physikalische Chemie 60: 399-412.

30. Kaufman, L., ed. 1975. Report on the proceedings of the fourth calphad meeting (August 18-22, 1975) National Birreau of Standards, Gaithersburg, Maryland.

31. Krupkowski, A. 1950. Activity coefficients in binary and many component solutions. Polska Akademia Umiejetnosci, Krakow, Poland, Bulletin International Supplement Series A, 1-4: 15-45.

32. LaFara, R. L. 1973. Computer methods for science and engineering. Hayden Book Company, Inc., Rochelle Park, N.J.

33. Lewis, G. N. and Randa11, M. 1961. Thermodynamics. Second Edition revised by K. S. Pitzer and L. Brewer. McGraw-Hill Book Company, New York, N.Y. 
34. Lumsden, J. 1952. Thermodynamics of alloys: Institute of Metals Monograph and Report Series 11: 206-234.

35. Lupis, C. H, P, 1968. On the definitions of Raoult's and Henry's 1aws. Scripta Metallurgica 2: 429-434.

36. Lupis, C. H. P. 1968. On the use of polynomials for the thermodynamics of dilute metallic solutions. Acta Metallurgica 16: 1365-1375.

37. Lupis, C. H. P. 1972. On the thermodynamic formalism of metaliic solutions. Pages $1-36$ in S. Z. Beer, ed. Liquid metals chemistry and physics. Marcel Dekker, Inc., New York, N.Y.

38. Lupis, C. H. P. and Elliott, J. F. 1965. Free energy, entropy, and enthalpy interaction coefficients. Transactions of the Metallurgical Society of AIME 233: 829830 .

39. Lupis, C. H. P. and E11iott, J. F. 1966. Generalized interaction coefficients, Part I: Definitions. Acta Metallurgica 14: 529-538.

40. Lupis, C. H. P. and E1liott, J. F. 1966. Generalized interaction coefficients, Part II: Free energy terms and the quasi-chemical theory. Acta Metallurgica 14: $1019-1032$.

41. Lupis, C.H. P. and Elliott, J. F. 1967. Prediction of enthalpy and entropy interaction coefficients by the "central atoms" theory. Acta Metallurgica 15: 265-276.

42. Margules, M. 1895. Über die Zusammensetzung der gesättigten Dämpe von Mischungen. Sitzungsberichte der Mathematisch--Naturwissenchaftlichen classe der Kaiser1 ichen Akademie der Wissenschaften 104, Abteilung 2a: 1243-1278.

43. Martin-Garin, R., Petot-Ervas, G., Petot, C. and Bonnier, E. 1969. Thermodynamique des systémes présentant une lacune de miscibilite. Journal de Chimie Physique et de Physico-Chimie Biologique 66: 1913-1918.

44. Mellor, J. W. 1955. Higher mathematics for students of chemistry and physics. Fourth edition. Dover Publications, Inc., New York, N.Y. 557-563.

45. Pelton, A. D. and Thompson, W. T. 1975. Phase diagrams. Progress in Solid-State Chemistry 10, Part 3: 119-155. 
46. Pennington, R. H. 1958. Introductory computer methods and numerical analysis. Second edition. McGraw-Hill Book Company, Inc. New York, N.Y.

47. Petot-Ervas, G., Petot, C., Desré, P. and Bonnier, É. 1968. No. 691--Correlations etre les propriétés thermodynamiques des systémes binaires et la géometrie des diagrammes de phases au voisinage du corps pur. Bulletin de Ia Societe chimique de France No. 11: 4335-4337.

48. Rao, M. V., Hiskes, R. and Tiller, W. A. 1973. Determination of solute interaction parameters by analysis of phase equilibria using a linear programming technique. Acta Metallurgica 21: 733-740.

49. Rollason, E. C. and Hysel, V. B. 1938. The constitution of lead-rich alloys of lead and cadmium. Journal of the Institute of Metals 63: 191-200.

50. Rudman, P. S. 1970. Thermodynamic analysis and synthesis of phase diagrams: I. Binary, substantially disordered, substitutional systems. Pages 147-292. In $\mathrm{H}$. Herman, ed. Advances in Materials Research. Vol. 4. Interscience Publishers, New York, N.Y.

51. Scatchard, G. and Hamer, W. J. 1935. The application of equations for the chemical potentials to partially miscible solutions. Journal of the American Chemical Soclety 57: 1805-1809.

52. Schenck, H. Frohberg, M. G. and Steinmetz, E. 1960. Ableitungen zur Begriffsbestimmung thermodynamischer Wirkungsgröben in Mehrstoffsystemen. Archiv für das Eisenhut tenwesen.31: 671-676.

53. Schleicher, A. P. 1912. Experimentelle untersuchungen am System Cd-Sn. Internationale Zeitschrift fur Metallographie.2: 76-89:

54. Schürmann, E. 1959. Zur quantitativen Thermischen Analyse. Auswertemöglichkeiten der quantitativen Thermischen Analyse von Zweistoffsystemen, angewand be $i$ den Warmeinhaltsmessungen der Blei-Kadmium-Legierungen. Archiv für das Eisenhüttenwesen 30: 103-121.

55. Shunk, F. A. 1969. Constitution of binary alloys. Second supplement. New York, N.Y., McGraw-Hill Book Company, Inc. 
56. Sigworth, G. K. and Elliott, J. F. 1974. The thermodynamics of liquid dilute iron alloys. Metal Science 8: $298-310$.

57. Simmons, M. F. 1966. Correlation of thermodynamic data with binary eutectic-type phase diagrams. Unpublished M.S. thesis. Library, Iowa State University of Science and Technology, Ames, IA.

58. Sundquist, B. E. 1966. The calculation of thermodynamic properties of miscibility-gap system. Transactions of the Metallurgical Society of AIME 236: 1111-1122.

59. Tammann, G. 1925. A text book of metallography. First Edition. The Chemical Catalog Company, Inc., New York, N.Y.

60. van Laar, J. J. 1910. Über Dampfspannungen von binären Gemischen. Zeitschrift fur Physikalische Chemie 72: $723-751$.

61. van der Toorn, L. J. and Tiedema, T. J. 1960. Precipitation in gold-platinum alloys--I Thermodynamics. Acta Metallurgica 8: 711-714.

62. Wagner, C. 1952. Thermodynamics of alloys. AddisonWesley Press, Inc., Cambridge, Mass.

63. Watson, W. A., Philipson, T. and Oates, P. G. 1969. Numerical analysis-the mathematics of computing. Vol. 2. American Elsevier Publishing Company, Inc., New York, N.Y.

64. Whittaker, E. and Robinson, G. 1967. The calculus of observations. Fourth edition. Dover Publications, Inc., New York, N.Y.

65. Williams, R. O. 1969. Series representation of thermodynamic functions of binary solutions. Transactions of the Metallurgical Society of AIME 245: 2565-2570.

66. Wilson, G. M. 1964. A new expression for the excess free energy of:mixing. Journal of the Americal Chemical Society 86: $127: 130$.

67. Wriedt, H. A. 1961. Calculation of activities in binary systems having miscibility gaps. Transactions of the Metallurgical Society of AIME 221: 377-383. 
VII. ACKNOWLEDGEMENTS

I would like to express special thanks to Dr. Premo Chiotti for his encouragement and guidance throughout this investigation.

I would also like to thank Dr. Robert J. Lambert, Dr. John W, Patterson, Marvin S. Beck, and John T. Mason for their helpful discussions concerning various aspects of this work.

I also wish to express special thanks to Miss Verna Thompson for typing this manuscript.

I wish to express my deepest appreciation to my parents for their love, encouragement and support throughout my graduate study. 


\section{APPENDIX}

A. Derivation of Equation I-10

The derivations presented in this section and in section VIII-B have been given by Chiotti (7). The previously discussed (see pages 26-27) Equation II-29,

$$
\Delta \bar{G}_{i}^{X S^{\prime}}=\Delta \bar{G}_{i}^{X S^{\prime \prime}}=R T \ln \frac{x_{i}^{\prime \prime}}{x_{i}^{\prime}}-\Delta G_{i}^{0, " \rightarrow \prime}
$$

is used as the basis for deriving Equations I-10, II-38, and II-39. Equation II-29 can be derived from the concept of the equality of chemical potentials for a component in two equilibrium phases.

For brevity, let $\Delta \bar{G}_{i}^{X S^{\prime}}=Y^{\prime}$, and $\Delta \bar{G}_{i}^{X S^{\prime \prime}}=Y^{\prime \prime}$. Also, unless otherwise specified, all of the variables are for component 1 , the solvent component. Incorporating these changes and inverting the argument of the logarithmic term, Equation II-29 can be rewritten as

$$
Y^{\prime}-Y^{\prime \prime}=-R T \ln \left(\frac{x^{\prime}}{x^{\prime \prime}}\right)-\Delta G^{0},^{\prime \prime}
$$

The following functional relationships can be stated for the variables in Equation VIII-1.

$$
\begin{aligned}
& Y^{\prime}=f\left(T, X^{\prime}\right) \quad T=f\left(X^{\prime}\right) \\
& Y^{\prime \prime}=f\left(T, x^{\prime \prime}\right) \quad T=f\left(x^{\prime \prime}\right) \\
& x^{\prime \prime}=f\left(x^{\prime}\right) \text { and } \quad \Delta G^{\circ},{ }^{\prime \prime}=f(T)
\end{aligned}
$$

The phase rule is given by $F=c-p+2$, where $F$ is the number of degrees of freedom; $c$ is the number of components 
and $\mathrm{p}$ is the number of phases. For a binary system at constant pressure with two phases in equilibrium, the phase rule dictates that only one degree of freedom exists and thus, that there can be only one independent variable. Fixing any one of the variables $T, x_{1}^{\prime}, x_{2}^{\prime}, x_{1}^{\prime \prime}$, or $x_{2}^{\prime \prime}$ fixes the state of the system. For the following derivations, $\ddot{x}_{1}^{\prime}$ is chosen as the independent variable.

In the limiting expressions to be given below, the following assumptions are implemented. The first assumption is that Raoult's law holds in the limit. Thus, $\left(\partial Y^{\prime} / d x^{\prime}\right)_{X^{\prime} \rightarrow 1}=0$, $\left(\partial Y^{\prime \prime} / \partial X^{\prime \prime}\right)_{X^{\prime \prime} \rightarrow 1}=0,\left(\partial \Delta \bar{S}_{1}^{X S} / \partial X^{\prime}\right)_{X^{\prime} \rightarrow 1}=0$, etc. The second assumption is that $\Delta \overline{\mathrm{S}}_{1}^{\mathrm{XS}}$ and $\Delta \overline{\mathrm{H}}_{1}^{\mathrm{XS}}$ are independent of temperature and thus, $\left(\partial \Delta \bar{S}_{1}^{X S} / \partial T\right)_{X^{\prime} \rightarrow 1}=0$.

Equation VIII-I is differentiated twice with respect to $x^{\prime}$ and the limit is taken of the resultant expression as $x^{i}+1\left(x^{\prime \prime} \rightarrow 1\right)$. The differentiation process is carried out below, term by term:

1) Differentiation of $Y^{\prime}=f\left(T, x^{\prime}\right)$ gives

$$
\left(\frac{\partial Y^{\prime}}{\partial x^{\prime}}\right)_{T}+\left(\frac{\partial Y^{\prime}}{\partial T}\right)_{x^{\prime}} \frac{d T}{d x^{\prime}}
$$

Differentiation of the expression a second time yields:

$$
\begin{gathered}
\left(\frac{\partial^{2} Y^{\prime}}{\partial x^{\prime 2}}\right)_{T}+\left(\frac{\partial\left[\partial Y^{\prime} / \partial x^{\prime}\right]}{\partial T}\right)_{x^{\prime}} \frac{d T}{d x^{\prime}}+\left(\frac{\partial^{2} Y^{\prime}}{\partial T^{2}}\right)_{x^{\prime}}\left(\frac{d T}{d x^{\prime}}\right)^{2} \\
+\left(\frac{\partial\left[\partial Y^{\prime} / \partial T X^{\prime}\right.}{\partial x^{\prime}}\right) \frac{d T}{d x^{\prime}}+\left(\frac{\partial Y^{\prime}}{\partial T}\right) \cdot \frac{d^{2} T}{x^{\prime}}
\end{gathered}
$$


Taking the limit as $x^{\prime}+1$ gives $\left(\partial^{2} Y^{\prime} / \partial X^{\prime 2}\right) T x^{\prime} \rightarrow 1$ with all the other terms going to zero.

2) Differentiating the term $-Y^{\prime \prime}=f\left(T, x^{\prime \prime}\right)$ with respect to $x^{\prime}$ gives:

$$
-\left(\frac{\partial Y^{\prime \prime}}{\partial x^{\prime \prime}}\right)_{T} \frac{d x^{\prime \prime}}{d x^{\prime}}-\left(\frac{\partial Y^{\prime \prime}}{\partial T}\right)_{x^{\prime \prime}}
$$

Differentiating this expression again yields,

$$
\begin{aligned}
-\left(\frac{\partial^{2} Y^{\prime \prime}}{\partial x^{\prime \prime}}\right)_{T}\left(\frac{d x^{\prime \prime}}{d x^{\prime}}\right)^{2}= & \left(\frac{\partial\left[\frac{\partial Y^{\prime \prime}}{\partial x^{\prime \prime}}\right]_{T}}{\partial T}\right)_{x^{\prime \prime}}\left(\frac{d x^{\prime \prime}}{d x^{\prime}}\right) \frac{d T}{d x^{\prime}}-\left(\frac{\partial^{2} Y^{\prime \prime}}{\partial T^{2}}\right)_{x^{\prime \prime}} \\
& -\left(\frac{\partial\left[\frac{\partial Y^{\prime \prime}}{\partial T}\right]^{\prime \prime}}{\partial x^{\prime \prime}}\right)_{T} \frac{d x^{\prime \prime}}{d x^{\prime}}
\end{aligned}
$$

Taking the limit as $x^{\prime}+1$, and $x^{\prime \prime} \rightarrow 1$ gives

$$
-\left[\left(\frac{\partial^{2} Y^{\prime \prime}}{\partial x^{\prime \prime 2}}\right)_{T}\left(\frac{d x^{\prime \prime}}{d x^{\prime}}\right)^{2}\right]_{\substack{x^{\prime} \rightarrow 1 \\ x^{\prime \prime} \rightarrow 1}}
$$

3) Differentiating the first term on the right side of Equation VIII-1 with respect to $x^{\prime}$, gives

$$
-\operatorname{RT}\left(\frac{\mathrm{d} \ln \frac{\mathrm{x}^{\prime}}{\mathrm{x}^{\prime \prime}}}{\mathrm{d} \mathrm{x}^{\prime}}\right)-\mathrm{R} \ln \frac{\mathrm{x}^{\prime}}{\mathrm{x}^{\prime \prime}}\left(\frac{\mathrm{dT}}{\mathrm{d} \mathrm{x}^{\prime}}\right)
$$

Differentiating the expression a second time yields

$$
-\operatorname{RI}\left(\frac{d^{2} \ln \frac{x^{\prime}}{x^{\prime \prime}}}{d x^{\prime 2}}\right)-2 R\left(\frac{d \ln \frac{x^{\prime}}{x^{\prime \prime}}}{d x^{\prime}}\right) \frac{d T}{d x^{\prime}}-R \ln \frac{x^{\prime}}{x^{\prime \prime}}\left(\frac{d^{2} T}{d x^{\prime 2}}\right)
$$

Taking the limit as $x^{1 \rightarrow 1}$ gives 


$$
-R T\left(\frac{d^{2} \ln \frac{x^{\prime}}{x^{\prime \prime}}}{d x^{\prime 2}}\right)_{x^{\prime} \rightarrow 1}-2 R\left(\frac{d \ln \frac{x^{\prime}}{x^{\prime \prime}}}{d x}\right)_{x^{\prime} \rightarrow 1}\left(\frac{d T}{d x^{\prime}}\right)_{x^{\prime} \rightarrow 1}
$$

4) Differentiating the last term of Equation VIII-1 results in

$$
=\frac{d\left(\Delta G^{0},{ }^{\prime \prime} \rightarrow^{\prime}\right)}{d T}\left(\frac{d T}{d x}\right)=\Delta S^{0,,^{\prime \prime \prime}}\left(\frac{d T}{d x}\right)
$$

Differentiating the expression again gives

$$
\Delta S^{0, " \rightarrow 1} \frac{d^{2} T}{d x^{\prime 2}}+\frac{d \Delta S^{0}, " \rightarrow 1}{d T}\left(\frac{d T}{d x^{\prime \prime}}\right)
$$

Assuming that $\Delta S^{O}, " \rightarrow$ is independent of temperature and taking the limit as $x^{\prime \rightarrow 1}$ gives

$$
\Delta S_{f}^{0}\left(\frac{d^{2} T}{d x^{\prime 2}}\right)_{x^{\prime} \rightarrow 1},
$$

$\Delta S^{0, " \rightarrow l}$ becoming $\Delta S_{f}^{o}$ in the limit.

Combining the limiting values from the steps $1,2,3$, and 4 gives

$$
\begin{aligned}
& \left(\frac{\partial^{2} Y^{\prime}}{\partial x^{\prime 2}}\right)_{T, x^{\prime} \rightarrow 1}-\left[\left(\frac{\partial^{2} Y^{\prime \prime}}{\partial x^{\prime 2}}\right)_{T}\left(\frac{d x^{\prime \prime}}{d x^{\prime}}\right)^{2}\right]_{\substack{X^{\prime} \rightarrow 1 \\
x^{\prime \prime} \rightarrow 1}}= \\
& -R T\left(\frac{d^{2} \ln \frac{x^{\prime}}{x^{\prime \prime}}}{d x^{\prime 2}}\right)_{x^{\prime} \rightarrow 1}-2 R\left(\frac{d \ln \frac{x^{\prime}}{x^{\prime \prime}}}{d x^{\prime}}\right)_{x^{\prime} \rightarrow 1}\left(\frac{d T}{d x}\right)_{x^{\prime} \rightarrow 1} \\
& +\Delta S^{0, " \rightarrow 1}\left(\frac{d^{2} T}{d x^{\prime 2}}\right)_{x^{\prime}+1}
\end{aligned}
$$


By differentiating Equation VIII-1 once with respect to $x^{\prime}$ and taking the limit as $x^{\prime} \rightarrow 1$, the following equality can be derived:

$$
\left(\frac{d x^{\prime \prime}}{d x^{\top}}\right)_{\substack{x^{\prime} \rightarrow 1 \\ x^{\prime \prime} \rightarrow 1}}=\left[1-\frac{\Delta S_{f}^{0}}{R T_{f}} \frac{d T}{d x^{\prime}}\right]_{\substack{x^{\prime} \rightarrow 1 \\ x^{\prime \prime} \rightarrow 1}}
$$

Chiotti (6) has obtained the following relationship from the Gibbs-Duhem equation:

$$
\left(\frac{\partial^{2} Y_{1}}{\partial x_{1}^{2}}\right)_{x_{1} \rightarrow 1}=-\left(\frac{\partial Y_{2}}{\partial x_{2} x_{1} \rightarrow 1}\right.
$$

Since

$$
\left(\frac{\partial Y_{2}}{\partial x_{2}}\right)_{\substack{T, P \\ x_{1} \rightarrow 1}}=\operatorname{RT}\left(\frac{\partial \ln \gamma_{2}}{\partial x_{2}}\right)_{\substack{x_{1} \rightarrow 1 \\ x_{2} \rightarrow 0}}=\operatorname{RT} \varepsilon_{2}^{2},
$$

Equation VIII-4 can be rewritten as

$$
\left(\frac{\partial^{2} Y_{1}}{\partial x_{1}^{2}}\right)_{x_{1} \rightarrow 1}=-R T \varepsilon_{2}^{2}
$$

Utilizing Equations VIII-3 and VIII-5, Equation VIII-2 can be rewritten as,

$$
\begin{aligned}
& \varepsilon_{2}^{2}+\left(\frac{\partial^{2} Y_{1}^{\prime \prime}}{\partial x_{1}^{\prime \prime}}\right)\left[1-\frac{\Delta S_{f}^{o}}{R T_{f}} \cdot \frac{d T}{d x_{1}^{\prime}}\right]=\frac{d^{2} \ln \frac{x_{1}^{1}}{x_{1}^{\prime \prime}}}{d x_{1}^{\prime 2}} \\
& +\frac{2}{T}\left(\frac{d \ln \frac{x_{1}^{\prime}}{x_{1}^{\prime \prime}}}{d x_{1}^{\prime}}\right) \frac{d T}{d x_{1}^{\prime}}-\left(\frac{\Delta S_{f}^{0}}{R T_{f}} \frac{d^{2} T}{d x_{1}^{\prime 2}}\right)
\end{aligned}
$$


where it is understood that all the terms in this expression are for the limiting case of $x^{\prime \rightarrow 1}$ and $x^{\prime \prime} \rightarrow 1$.

To obtain Equation I-10, it is assumed that the second term on the left side of Equation VIII- 6 is negligibie. Substitution of various liquidus values of $\left(\mathrm{dT} / \mathrm{dx} \mathrm{l}_{1}^{\prime}\right)$ into this term indicate that the value of $\left[\left(\partial^{2} Y_{1}^{\prime \prime}\right) /\left(\partial x_{1}^{\prime \prime 2}\right)\right]_{T}$ would have to be exceedingly large for the term to make a significant contribution to the equation. Assuming this term to be zero, Equation $1-10$ follows:

$$
\begin{aligned}
& \varepsilon_{2}^{2}=\left(\frac{\mathrm{d}^{2} \ln \frac{\mathrm{x}_{1}^{\prime}}{\mathrm{x}_{1}^{\prime \prime}}}{\mathrm{d} \mathrm{x}_{1}^{\prime 2}}\right)_{\mathrm{x}_{1}^{\prime} \rightarrow 1}+\frac{2}{\mathrm{~T}_{\mathrm{f}_{1}}}\left(\frac{\mathrm{d} \ln \frac{\mathrm{x}_{1}^{\prime}}{\mathrm{x}_{1}^{\prime \prime}}}{\mathrm{d} \mathrm{x}_{1}^{\prime}}\right)_{\mathrm{x}_{1}^{\prime} \rightarrow 1}\left(\frac{\mathrm{d} T}{\mathrm{dx}}\right)_{\mathrm{x}_{1}^{\prime} \rightarrow 1} \\
& -\frac{\Delta S_{f_{1}}^{o}}{R T_{f_{1}}}\left(\frac{d^{2} T}{d x_{1}^{2}}\right)_{x_{1}^{\prime}+1}
\end{aligned}
$$

B. Derivation of Equations II-38 and II-39

Equation II-38, the expression which is equivalent to the expression derived by Petot-Ervas et al. (47), can be derived by the following alterations in the procedure given in section VIII-A. In step 3 of the above procedure, instead of leaving the logarithm terms in the form:

$$
\frac{d \ln \frac{x^{\prime}}{x^{\prime \prime}}}{d x^{\prime}},
$$

the differentiation is carried out completely. Taking the 
first derivative of the first term on the right side of Equation VIII-1 gives:

$$
-\frac{R T}{x^{\prime}}-R \ln x^{\prime}\left(\frac{d T}{d x^{\prime}}\right)+\frac{R T}{x^{\prime \prime}}\left(\frac{d x^{\prime \prime}}{d x}\right)+R \text { ln } x^{\prime \prime}\left(\frac{d T}{d x}{ }^{\prime}\right)
$$

Differentiating again with respect to $x^{\prime}$ and taking the limit as $x^{\prime \rightarrow 1}$ and $x^{\prime \prime \rightarrow 1}$ yields

$$
\left[R T-2 R\left(\frac{d T}{d x^{\top}}\right)-R T\left(\frac{d x^{\prime \prime}}{d x^{\prime}}\right)^{2}+R T\left(\frac{d^{2} x^{\prime \prime}}{d x^{\prime}}\right)+2 R \frac{d x^{\prime \prime}}{d x^{\prime}}\left(\frac{d T}{d x^{\prime}}\right)\right]_{\substack{x^{\prime}+1 \\ x^{\prime \prime} \rightarrow 1}}
$$

As before, the limiting expression from step 1 gives

$$
\left(\frac{\partial^{2} Y^{\prime}}{\partial x^{\prime 2}}\right)_{X^{\prime} \rightarrow 1}=-R T \varepsilon_{2}^{2},^{\prime}
$$

Using the nomenclature of Petot-Ervas et al.,

$$
\rho^{0} \equiv\left(\frac{d x^{\prime \prime}}{d x}\right)_{\begin{array}{l}
x^{\prime} \rightarrow 0 \\
x^{\prime \prime} \rightarrow 0
\end{array}}
$$

Using Equation VIII-5 for the solid phase gives:

$$
\left(\frac{\partial^{2} Y^{\prime \prime}}{\partial x^{\prime \prime 2}}\right)_{x_{1}^{\prime \prime} \rightarrow 1}=-R T \varepsilon_{2}^{2}, " \text {. }
$$

The limiting expression for step 2 is then,

$$
+\mathrm{RT} \varepsilon_{2}^{2}, " \rho 02
$$

Incorporating these alterations and combining the limiting expressions for steps $1,2,3$, and 4 gives: 


$$
\begin{aligned}
& -\varepsilon_{2}^{2},{ }^{\prime}+\rho^{\circ 2} \varepsilon_{2}^{2}, "=\left(1-\rho^{\circ 2}\right)-\frac{2}{T}\left(1-\rho^{\circ}\right)\left(\frac{d T}{d x_{1}^{\prime}}\right)+\left(\frac{d^{2} x_{1}^{\prime \prime}}{d x_{1}^{\prime 2}}\right) \\
& +\frac{\Delta S_{f_{1}}^{0}}{R T_{f_{1}}}\left(\frac{d^{2} T}{d x_{1}^{\prime 2}}\right)
\end{aligned}
$$

Applying Equation VIII-3 to the seciond term on the right side of Equation VII 8 gives Equation II -38 :

$$
\begin{aligned}
& -\varepsilon_{2}^{2},+\rho^{\circ} \varepsilon_{2}^{2}, "=\left(1-\rho^{o^{2}}\right)-\frac{2 R}{\Delta S_{f_{1}}^{o}}\left(1-\rho^{\circ}\right)^{2}+\left(\frac{d^{2} x_{1}^{\prime \prime}}{d x_{1}^{\prime 2}}\right) \\
& +\frac{\Delta S_{f_{1}}^{o}}{R T_{f_{1}}}\left(\frac{d^{2} T}{d x_{1}^{\prime 2}}\right)
\end{aligned}
$$

If the solid solubility is negligible, i.e., $x^{\prime \prime}=1$ for all $T$, then

$$
\rho^{0}=\left(\frac{d x^{\prime \prime}}{d x^{\prime}}\right)_{\substack{x^{\prime} \rightarrow 1 \\ x^{\prime \prime} \rightarrow 1}}=0 \text { and }\left(\frac{d^{2} x^{\prime \prime}}{d x^{\prime 2}}\right)_{x^{\prime} \rightarrow 1}=0
$$

Equation VII=9 simplified immediately to Equation. II-39:

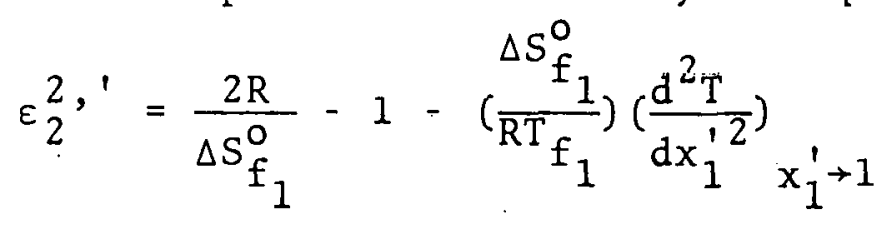

